REPRESENTATION THEORY

An Electronic Journal of the American Mathematical Society

Volume 15, Pages 407-432 (May 16, 2011)

S 1088-4165(2011)00395-2

\title{
COMPATIBLE INTERTWINERS FOR REPRESENTATIONS OF FINITE NILPOTENT GROUPS
}

\author{
MASOUD KAMGARPOUR AND TERUJI THOMAS
}

\begin{abstract}
We sharpen the orbit method for finite groups of small nilpotence class by associating representations to functionals on the corresponding Lie rings. This amounts to describing compatible intertwiners between representations parameterized by an additional choice of polarization. Our construction is motivated by the theory of the linearized Weil representation of the symplectic group. In particular, we provide generalizations of the Maslov index and the determinant functor to the context of finite abelian groups.
\end{abstract}

\section{INTRODUCTION}

This paper is about the complex irreducible representations of finite nilpotent groups $G$ of small nilpotence class. Here small means 'strictly smaller than any prime divisor of $|G|$ '. However, it is convenient to remember that any finite nilpotent group canonically decomposes as the direct product of its Sylow subgroups, so that we can henceforce assume that $G$ is a finite $p$-group of nilpotence class strictly less than $p$. We will also assume that $p$ is an odd prime, since $p=2$ forces $G$ to be abelian.

In general terms, our goal is to associate to $G$ a canonical abelian group $\mathfrak{g}^{*}$, and to each $f \in \mathfrak{g}^{*}$ a canonical representation $\rho_{f}$ of $G$. $G$ will act on the right of $\mathfrak{g}^{*}$, the isomorphism class of $\rho_{f}$ will depend only on the orbit of $f$ (see Remark 1.3 below), and this association between orbits and isomorphism classes will be bijective.

Remark 1.1. When $G$ is abelian, one can take $\mathfrak{g}^{*}$ to be the Pontryagin dual $\mathfrak{g}^{*}=$ $\operatorname{Hom}\left(G, \mathbb{C}^{\times}\right)$, with the trivial action of $G$. Of course, $\rho_{f}$ is just $f$ considered as a one-dimensional representation of $G$.

Remark 1.2. In saying that $\mathfrak{g}^{*}$ and the function $f \mapsto \rho_{f}$ are 'canonical', we mean, at least, that they vary functorially with isomorphisms between groups $G$ : to each isomorphism $\alpha: G_{1} \rightarrow G_{2}$ of groups, there corresponds an isomorphism $\alpha^{*}: \mathfrak{g}_{2}^{*} \rightarrow$ $\mathfrak{g}_{1}^{*}$ of abelian groups and, for each $f \in \mathfrak{g}_{2}^{*}$, an isomorphism $\rho_{\alpha^{*} f} \rightarrow \rho_{f} \circ \alpha$ of representations of $G_{1}$.

Received by the editors October 29, 2009 and, in revised form, August 16, 2010.

2010 Mathematics Subject Classification. Primary 20C15.

Key words and phrases. Orbit method, p-groups, neighboring polarizations, Lie rings, intertwiners, Weil representation, determinant, orientation, Maslov index, determinant of abelian groups.

The first author was supported by NSERC PDF grant. The second author was supported by a JRF at Merton College, Oxford and a Seggie Brown Fellowship at Edinburgh.

(C)2011 American Mathematical Society Reverts to public domain 28 years from publication 
Remark 1.3. The given right action of $G$ on $\mathfrak{g}^{*}$ corresponds functorially to the left action Ad of $G$ on itself by conjugation. By Remark 1.2, we also obtain an isomorphism $\rho_{\mathrm{Ad}^{*}(g) f} \rightarrow \rho_{f} \circ \operatorname{Ad}(g)$ for each $g \in G$ : we can say that $f \mapsto \rho_{f}$ is $G$-equivariant. This makes precise the statement that $\rho_{f}$ depends only on the orbit of $f$.

1.1. Precise statement of the problem. Let us now describe the background to our work and the specific question it answers. In [BD06, §2], M. Boyarchenko and V. Drinfeld have modified Kirillov's orbit method Kir62 to describe irreducible representations of $G$ (see also [How77, §6], and [Kaz77]). The construction works as follows (with more details in $\S ₫ 2 \sqrt{3}$ ).

To $G$ one associates a Lie ring, that is to say, an abelian group $\mathfrak{g}$ equipped with a bracket $[\cdot, \cdot]$ satisfying the Jacobi identity and $[x, x]=0$ for all $x \in \mathfrak{g}$. The underlying set of $\mathfrak{g}$ equals that of $G$. The relationship between $\mathfrak{g}$ and $G$ is analogous to that of Lie algebras and Lie groups. The multiplication in $G$ is given by the usual Campbell-Hausdorff formula:

$$
x * y=x+y+\frac{[x, y]}{2}+\text { (higher order terms) } .
$$

There is a one-to-one correspondence between subgroups of $G$ and subrings of $\mathfrak{g}$. Furthermore, one can define the adjoint action of $G$ on $\mathfrak{g}$ in an obvious way. We set $\mathfrak{g}^{*}:=\operatorname{Hom}\left(\mathfrak{g}, \mathbb{C}^{\times}\right)$.

Pick $f \in \mathfrak{g}^{*}$. A polarization for $f$ is a subring $\mathfrak{p} \subseteq \mathfrak{g}$ satisfying $f([\mathfrak{p}, \mathfrak{p}])=\{1\}$, such that $\mathfrak{p}$ is maximal among all additive subgroups of $\mathfrak{g}$ with this property (cf. Theorem 2.4). Let $P$ be the subgroup of $G$ corresponding to a polarization $\mathfrak{p}$. It follows from (1.1) that $f$ defines a homomorphism $\chi_{f}: P \rightarrow \mathbb{C}^{\times}$. The orbit method states that $\rho_{f, \mathfrak{p}}:=\operatorname{ind}_{P}^{G} \chi_{f}$ is an irreducible representation of $G$. Furthermore, the isomorphism class of $\rho_{f, \mathfrak{p}}$ depends only on the coadjoint orbit of $f$, and every isomorphism class arises in this way.

1.1.1. The main goal of this article is to sharpen the orbit method by constructing a canonical representation $\rho_{f}$ of $G$ for each $f \in \mathfrak{g}^{*}$, independent of the choice of polarization. We do this by constructing compatible intertwiners between the various representations $\rho_{f, \mathfrak{p}}$, as we now explain.

1.1.2. The intertwiner between $\rho_{f, \mathfrak{p}_{1}}$ and $\rho_{f, \mathfrak{p}_{2}}$ will depend on a choice of orientations of $\mathfrak{p}_{1}$ and $\mathfrak{p}_{2}$. An orientation of a vector space $V$ is a nonzero element 1 of the determinant (highest exterior power) $\operatorname{det}(V)$; in Appendix A, we develop the corresponding theory of determinants of finite abelian $p$-groups. We can then define an oriented polarization to be a pair $\tilde{\mathfrak{p}}=(\mathfrak{p}, o)$ consisting of a polarization $\mathfrak{p}$ and a nonzero element $o \in \operatorname{det}(\mathfrak{p})$.

Thus, for every pair $\tilde{\mathfrak{p}}_{1}, \tilde{\mathfrak{p}}_{2}$ of oriented polarizations, we describe intertwining operators $\Psi_{\tilde{\mathfrak{p}}_{1}, \tilde{\mathfrak{p}}_{2}} \in \operatorname{Hom}_{G}\left(\rho_{f, \mathfrak{p}_{2}}, \rho_{f, \mathfrak{p}_{1}}\right)$ that are compatible; i.e., for any three oriented

\footnotetext{
${ }^{1}$ In the literature it is usual, and even here it would suffice, to identify two orientations $o_{1}$ and $o_{2}$ if $o_{1}=a^{2} o_{2}$ for some $a$ in the ground field. In that sense, for example, any real vector space has two orientations. In GH08, the less picturesque term 'enhancement' is used in place of what we call orientation.
} 
polarizations $\tilde{\mathfrak{p}}_{1}, \tilde{\mathfrak{p}}_{2}, \tilde{\mathfrak{p}}_{3}$, the following diagram commutes:

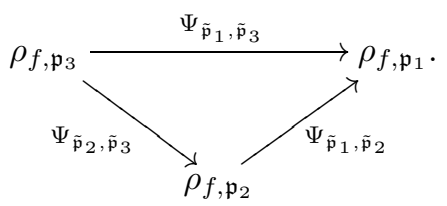

Let $\Lambda_{f}$ be the set of all oriented polarizations of $f$, and write $V_{f, \mathfrak{p}}$ for the representation space of $\rho_{f, \mathfrak{p}}$. Then the desired $\rho_{f}$ is the diagonal representation of $G$ on

$$
V_{f}:=\left\{\left(v_{\tilde{\mathfrak{p}}}\right) \in \bigoplus_{\tilde{\mathfrak{p}} \in \Lambda_{f}} V_{f, \mathfrak{p}} \mid v_{\tilde{\mathfrak{p}}_{1}}=\Psi_{\tilde{\mathfrak{p}}_{1}, \tilde{\mathfrak{p}}_{2}}\left(v_{\tilde{\mathfrak{p}}_{2}}\right) \text { for all } \tilde{\mathfrak{p}}_{1}, \tilde{\mathfrak{p}}_{2} \in \Lambda_{f}\right\}
$$

The projection of $V_{f}$ to each $V_{f, \mathfrak{p}}$ is an isomorphism between representations of $G$.

Remark 1.4. G. Lion and P. Perrin LP81] studied the same problem of constructing compatible intertwiners, but for unipotent algebraic groups defined over local fields. There is a crucial difference: in that setting one can only construct intertwiners that are compatible up to a sign. Nonetheless, we adapt several important ideas from their work; see Remark 1.8 and 1.4 for further comments.

1.1.3. Relationship to the Weil representation. Let $(A, \omega)$ be a symplectic vector space over $\mathbb{F}_{q}$. The Heisenberg group $H$ is the set $A \times \mathbb{F}_{q}$ equipped with the multiplication

$$
(v, t) *\left(v^{\prime}, t^{\prime}\right)=\left(v+v^{\prime}, t+t^{\prime}+\frac{1}{2} \omega\left(v, v^{\prime}\right)\right) .
$$

For example, if $A$ is two-dimensional, then $H$ is isomorphic to the group of $3 \times 3$ unipotent upper triangular matrices. The existence of compatible intertwiners for representations of $H$ is closely related to the fact that the projective Weil representation of the symplectic group $\operatorname{Sp}(A)$ can be linearized; cf. Wei64, Gér77, Per81, Kam05, GH07, and especially GH08. That is, for generic choices of $f$, the automorphism group of $(H, f)$ is isomorphic to $\operatorname{Sp}(A)$, and the resulting action of $\operatorname{Sp}(A)$ on $\Lambda_{f}$ lifts to a representation of $\operatorname{Sp}(A)$ on $V_{f}$. This is the Weil representation. The present work is essentially an extension of this theory to the setting of more general finite nilpotent groups.

1.2. Formulas for the intertwiners. The representation space $V_{f, \mathfrak{p}}$ is the space of functions $\phi: G \rightarrow \mathbb{C}$ satisfying

$$
\phi(p g)=\chi_{f}(p) \phi(g)
$$

for all $g \in G$ and $p \in P$. The representation $\rho_{f, \mathfrak{p}}$ is given by

$$
\left(\rho_{f, \mathfrak{p}}(h) \phi\right)(g)=\phi(g h) .
$$

It is unitary with respect to the Hermitian inner product

$$
\left(\phi_{1}, \phi_{2}\right)=\sum_{g \in P \backslash G} \overline{\phi_{1}(g)} \phi_{2}(g)
$$

on $V_{f, \mathfrak{p}}$. The averaging operator $\Phi_{\mathfrak{p}_{1}, \mathfrak{p}_{2}}$ defined by 2

$$
\left(\Phi_{\mathfrak{p}_{1}, \mathfrak{p}_{2}} \phi\right)(g):=\frac{1}{\sqrt{\left|\mathfrak{p}_{1}\right|\left|\mathfrak{p}_{1} \cap \mathfrak{p}_{2}\right|}} \sum_{p_{1} \in P_{1}} \chi_{f}^{-1}\left(p_{1}\right) \phi\left(p_{1} g\right)
$$

\footnotetext{
${ }^{2}$ Here and throughout this paper, we only use the symbol $\sqrt{ }$ to take positive square roots of positive real numbers.
} 
is a unitary operator in $\operatorname{Hom}_{G}\left(V_{f, \mathfrak{p}_{2}}, V_{f, \mathfrak{p}_{1}}\right)$. Moreover, $\Phi_{\mathfrak{p}_{1}, \mathfrak{p}_{2}}=\Phi_{\mathfrak{p}_{2}, \mathfrak{p}_{1}}^{-1}$.

However, these intertwiners are not compatible: by Schur's lemma there is a scalar $\alpha\left(\mathfrak{p}_{1}, \mathfrak{p}_{2}, \mathfrak{p}_{3}\right)$ of modulus 1 such that

$$
\Phi_{\mathfrak{p}_{1}, \mathfrak{p}_{2}} \circ \Phi_{\mathfrak{p}_{2}, \mathfrak{p}_{3}} \circ \Phi_{\mathfrak{p}_{3}, \mathfrak{p}_{1}}=\alpha\left(\mathfrak{p}_{1}, \mathfrak{p}_{2}, \mathfrak{p}_{3}\right) \mathrm{Id},
$$

but in general $\alpha$ does not equal 1 .

Guided by the case of the Heisenberg group [Per81, LV80] and the work of Lion and Perrin [LP81, we modify each $\Phi_{\mathfrak{p}_{1}, \mathfrak{p}_{2}}$ by a scalar so that the resulting operators are compatible. As explained in 1.1 .2 these scalars depend on a choice of orientations. To a pair $\tilde{\mathfrak{p}}_{1}, \tilde{\mathfrak{p}}_{2}$ of oriented polarizations, we associate (2.4) a relative orientation $\theta\left(\tilde{\mathfrak{p}}_{1}, \tilde{\mathfrak{p}}_{2}\right) \in \mathbb{F}_{p}^{\times} /\left(\mathbb{F}_{p}^{\times}\right)^{2}$. Let

$$
\Psi_{\tilde{\mathfrak{p}}_{1}, \tilde{\mathfrak{p}}_{2}}:=\gamma(1)^{-m\left(\mathfrak{p}_{1}, \mathfrak{p}_{2}\right)^{2}} \gamma\left(\theta\left(\tilde{\mathfrak{p}}_{1}, \tilde{\mathfrak{p}}_{2}\right)\right) \Phi_{\mathfrak{p}_{1}, \mathfrak{p}_{2}},
$$

where $m\left(\mathfrak{p}_{1}, \mathfrak{p}_{2}\right)=\log _{p}\left|\mathfrak{p}_{1}\right|-\log _{p}\left|\mathfrak{p}_{1} \cap \mathfrak{p}_{1}\right|-1$ and

$$
\gamma(a):=\sum_{x \in \mathbb{F}_{p}^{\times}} e^{i \pi a x^{2} / p} .
$$

Here is the main result of this paper.

Theorem 1.5. The operators $\left\{\Psi_{\tilde{\mathfrak{p}}_{1}, \tilde{\mathfrak{p}}_{2}}\right\}$ are compatible; i.e.,

$$
\Psi_{\tilde{\mathfrak{p}}_{1}, \tilde{\mathfrak{p}}_{2}} \circ \Psi_{\tilde{\mathfrak{p}}_{2}, \tilde{\mathfrak{p}}_{3}} \circ \Psi_{\tilde{\mathfrak{p}}_{3}, \tilde{\mathfrak{p}}_{1}}=\mathrm{Id}
$$

for any oriented polarizations $\tilde{\mathfrak{p}}_{1}, \tilde{\mathfrak{p}}_{2}, \tilde{\mathfrak{p}}_{3}$ of $f$.

Remark 1.6. The definitions of $\theta$ and $\gamma$ depend implicitly on the choice of a primitive $p^{\text {th }}$ root of unity. However, the intertwiner (1.5) is independent of this choice.

1.3. Reduction to the Heisenberg case. To prove Theorem 1.5 we first show that the cocycle $\alpha$ of (1.4) can be computed in 'Heisenberg terms'. Our goal now is to formulate this as a precise statement, to be proved in 4 . An analysis of $\alpha$ for the Heisenberg group will then yield Theorem 1.5.

Consider $\mathfrak{g}$ as an abelian group equipped with a skew-symmetric pairing $B_{f}(x, y):=f([x, y])$. Let $\mathfrak{z} \subset \mathbb{C}^{\times}$be the image of $B_{f}$. Note that every polarization of $f$ contains $\operatorname{Ker} B_{f}$. Make the abelian group $\mathfrak{g}^{f}:=\left(\mathfrak{g} / \operatorname{Ker} B_{f}\right) \oplus \mathfrak{z}$ into a Lie ring using the usual Heisenberg commutator; namely, $\mathfrak{z}$ is central and the bracket of $x, y \in \mathfrak{g} / \operatorname{Ker} B_{f}$ equals $B_{f}(x, y) \in \mathfrak{z}$.

Let $\bar{f}: \mathfrak{g}^{f} \rightarrow \mathbb{C}^{\times}$be the composition $\mathfrak{g}^{f} \rightarrow \mathfrak{z} \hookrightarrow \mathbb{C}^{\times}$. If $\mathfrak{p} \subseteq \mathfrak{g}$ is a polarization for $f$, then one can show that $\mathfrak{p}^{f}:=\left(\mathfrak{p} / \operatorname{Ker} B_{f}\right) \oplus \mathfrak{z}$ is a polarization for $\bar{f}$. Using $\left(\mathfrak{g}^{f}, \bar{f}\right)$ instead of $(\mathfrak{g}, f)$, we can define $\alpha\left(\mathfrak{p}_{1}^{f}, \mathfrak{p}_{2}^{f}, \mathfrak{p}_{3}^{f}\right)$. The main technical result is:

Theorem 1.7. For all polarizations $\mathfrak{p}_{1}, \mathfrak{p}_{2}, \mathfrak{p}_{3}$ of $f$,

$$
\alpha\left(\mathfrak{p}_{1}, \mathfrak{p}_{2}, \mathfrak{p}_{3}\right)=\alpha\left(\mathfrak{p}_{1}^{f}, \mathfrak{p}_{2}^{f}, \mathfrak{p}_{3}^{f}\right) .
$$

To prove this theorem we define, following a suggestion of Drinfeld, the notion of neighboring polarizations in 2.3. This is the key definition of this paper. We will show that any two polarizations can be connected by a chain of neighbors. Furthermore, the identity (1.7) is not hard to verify when $\mathfrak{p}_{1}$ and $\mathfrak{p}_{2}$ are neighbors (while $\mathfrak{p}_{3}$ is arbitrary). We complete the proofs of Theorem 1.7 and Theorem 1.5 in $\$ 4$ and $\$ 5$ respectively. 
Remark 1.8. Lion and Perrin (see Remark 1.4) proved an equality similar to (1.7) for unipotent groups over local fields. Using the present notion of neighboring polarizations, one can give a more transparent proof of their result.

Remark 1.9. R. Howe [How73] also studied automorphisms and representations of $p$-groups by reducing (in a somewhat different way) to the action of a symplectic group on a Heisenberg group.

1.4. Two constructions of independent interest. In Appendices $\mathrm{A}$ and $\mathrm{B}$ we generalize some ideas from linear algebra to the context of finite abelian $p$-groups. While the application of these ideas was inspired by [LP81, their development for finite groups appears to be new, and may be of independent interest.

1.4.1. Determinants. As explained in $\$ 1.1 .2$, we need a theory of determinants of finite abelian $p$-groups. In Appendix $\mathrm{A}$, we define and study the determinant functor in two complementary ways. In one approach, following a suggestion of Drinfeld, we define the determinant as the universal additive functor, in the sense of Deligne [Del87, §4], and then use Quillen's results on $K$-theory Qui73 to identify its target as the category of graded one-dimensional vector spaces over $\mathbb{F}_{p}$. In A.5. we sketch a more elementary approach using composition series and a form of the Jordan-Hölder theorem.

1.4.2. The Maslov index. In Appendix B, we adapt the theory of the Witt group and the Maslov index to the current setting (these notions are again widely known (see e.g. [LV80) for vector spaces rather than finite groups). In particular, in Proposition B.20, we use oriented Lagrangians to present the Maslov index as a coboundary. This is not possible over local fields (cf. Remark 1.4), and it plays a crucial role in proving Theorem 1.5 .

1.5. Organization of the text. In $\$ 2$ we set out the basic facts about Lie rings, polarizations, orientations, and examine the case of Heisenberg Lie rings. We define the notions of neighboring polarizations and relative orientation. In $\$ 3$, we recall in greater detail the orbit method mentioned in \$1.1 including the construction due to M. Lazard which defines an equivalence between the relevant categories of nilpotent Lie rings and groups. In $₫ 4$ we develop the reduction process of $\$ 1.3$ and prove Theorem 1.7. In $\$ 5$ we calculate the cocycle (1.4) in the Heisenberg case in terms of the Maslov index (Appendix B), and use the result to prove Theorem 1.5.

In Appendices $\mathrm{A}$ and $\mathrm{B}$, we develop the ideas of 1.4 . In Appendix $\mathrm{C}$ we collect the proofs of some minor results stated in the main text.

\subsection{Conventions.}

1.6.1. We fix $p$, an odd prime; all the groups, rings, and other structures that appear in this work are finite and of order a power of $p$.

1.6.2. For such a group $A$, we set $\operatorname{len}(A):=\log _{p}|A|$. If $A$ and $B$ are abelian groups, then $A \otimes B$ denotes the tensor product in the sense of $\mathbb{Z}$-modules, so a group homomorphism from $A \otimes B$ is the same thing as a bi-additive map from the set $A \times B$.

1.6.3. We fix a homomorphism $\psi_{0}: \mathbb{F}_{p} \rightarrow \mathbb{C}^{\times}$identifying the additive group $\mathbb{F}_{p}$ with the multiplicative group of $p$ th roots of unity. (The final results are independent of this choice; see Remark 1.6.) 
1.6.4. Finally, we adopt the following nonstandard sign rule, which causes some (usually simplifying) discrepancies from the literature. For any vector space $V$ of dimension $n$, we wish to fix an isomorphism

$$
\iota: \wedge^{n}\left(V^{*}\right) \stackrel{\sim}{\rightarrow}\left(\wedge^{n} V\right)^{*} .
$$

Our $\iota$ differs by a sign $(-1)^{n(n-1) / 2}$ from the standard one (see, e.g., Gai07, §5.1]). Concretely, it is determined by the following rule. Suppose that $e_{1}, \ldots, e_{n}$ and $e_{1}^{*}, \cdots, e_{n}^{*}$ are dual bases for $V$ and $V^{*}$. Then we require that $e_{1} \wedge \cdots \wedge e_{n}$ and $\iota\left(e_{n}^{*} \wedge \cdots \wedge e_{1}^{*}\right)$ are dual bases for $\wedge^{n} V$ and $\left(\wedge^{n} V\right)^{*}$. Here, as usual, $e_{1} \wedge \cdots \wedge e_{n}$ denotes the image of $e_{1} \otimes \cdots \otimes e_{n}$ under the canonical quotient $V^{\otimes n} \rightarrow \wedge^{n} V$.

\section{LIE RINGS AND POLARIZATIONS}

After laying out the general theory in $\$ \$ 2.12 .4$ we will work out the fundamental example (the Heisenberg Lie ring) in $\$ 2.5$.

\subsection{Lie rings.}

Definition 2.1. A Lie ring is an abelian group $\mathfrak{g}$ equipped with a bracket [., .]: $\mathfrak{g} \otimes$ $\mathfrak{g} \rightarrow \mathfrak{g}$ satisfying the Jacobi identity and the identity $[x, x]=0$.

Definition 2.2. The (Pontryagin) dual of $\mathfrak{g}$ is the abelian group $\mathfrak{g}^{*}:=\operatorname{Hom}\left(\mathfrak{g}, \mathbb{C}^{\times}\right)$. (Note that the bracket of $\mathfrak{g}$ does not enter into the definition of $\mathfrak{g}^{*}$.)

2.2. Polarizations. Henceforth $\mathfrak{g}$ denotes a finite nilpotent Lie ring.

Definition 2.3. A polarization of $f \in \mathfrak{g}^{*}$ is a Lie subring of $\mathfrak{p} \subseteq \mathfrak{g}$ such that $f([\mathfrak{p}, \mathfrak{p}])=\{1\}$ and $\mathfrak{p}$ is maximal among all subgroups of $\mathfrak{g}$ with this property.

Using the terminology of $\$$ B.1 , one can say that a polarization is a Lie subring that is Lagrangian for the skew pairing

$$
B_{f}(x, y):=f([x, y])
$$

on $\mathfrak{g}$. In particular, every polarization of $f$ contains $\operatorname{Ker}\left(B_{f}\right)$.

From the definition, it is not clear that a functional $f \in \mathfrak{g}^{*}$ has a polarization. The following theorem of A. Kirillov guarantees the existence of polarizations (see also, BD06], thm. 2.9 and app. D)

Theorem 2.4 ([Kir62]). Every $f \in \mathfrak{g}^{*}$ has a polarization.

2.3. Neighboring polarizations. Here is one of the key definitions of this paper.

Definition 2.5. Let $\mathfrak{p}_{1}$ and $\mathfrak{p}_{2}$ be polarizations for $f \in \mathfrak{g}^{*}$. We say $\mathfrak{p}_{1}$ and $\mathfrak{p}_{2}$ are neighbors if $\left[\mathfrak{p}_{1}, \mathfrak{p}_{2}\right] \subseteq \mathfrak{p}_{1} \cap \mathfrak{p}_{2}$ (in other words, if $\mathfrak{p}_{1}$ and $\mathfrak{p}_{2}$ normalize one another).

A chain connecting $\mathfrak{p}_{1}$ and $\mathfrak{p}_{2}$ is a finite sequence $\mathfrak{p}_{1}=\mathfrak{q}_{1}, \mathfrak{q}_{2}, \ldots, \mathfrak{q}_{m}=\mathfrak{p}_{2}$ of polarizations such that $\mathfrak{q}_{i}$ and $\mathfrak{q}_{i+1}$ are neighbors for every $i<m$.

Lemma 2.6. Let $\mathfrak{p}_{1}$ and $\mathfrak{p}_{2}$ be polarizations for $f \in \mathfrak{g}^{*}$. Then there exists a chain connecting them.

The proof is given in 


\subsection{Relative orientation of oriented polarizations.}

Definition 2.7. An orientation of a finite abelian $p$-group $A$ is a nonzero element $o \in \operatorname{det}(A)$ (see Appendix $\mathrm{A}$ ). An oriented abelian group is a pair $(A, o)$ where $o$ is an orientation of $A$.

To a pair of oriented polarizations $\tilde{\mathfrak{p}}_{i}=\left(\mathfrak{p}_{i}, o_{i}\right), i=1,2$, we associate an element $\theta\left(\tilde{\mathfrak{p}}_{1}, \tilde{\mathfrak{p}}_{2}\right) \in \mathbb{F}_{p}^{\times} /\left(\mathbb{F}_{p}^{\times}\right)^{2}$, called the relative orientation, in the following way. Let $\mathfrak{l}_{i}:=\mathfrak{p}_{i} /\left(\mathfrak{p}_{1} \cap \mathfrak{p}_{2}\right)$. Since Det is an additive functor (Definitions A.1 and A.3), we have isomorphisms

$$
\varphi_{i}: \operatorname{det}\left(\mathfrak{p}_{i}\right) \stackrel{\simeq}{\longrightarrow} \operatorname{det}\left(\mathfrak{l}_{i}\right) \otimes \operatorname{det}\left(\mathfrak{p}_{1} \cap \mathfrak{p}_{2}\right) .
$$

Choose an orientation $\bar{o}$ for $\mathfrak{p}_{1} \cap \mathfrak{p}_{2}$, and let $\bar{o}_{i}$ denote the orientation of $\mathfrak{l}_{i}$ satisfying $\varphi_{i}\left(o_{i}\right)=\bar{o}_{i} \otimes \bar{o}$. The pairing

$$
B: \mathfrak{p}_{1} \otimes \mathfrak{p}_{2} \rightarrow \mathbb{C}^{\times}, \quad(x, y) \mapsto f([x, y])
$$

induces a perfect 3 pairing $\mathfrak{l}_{1} \otimes \mathfrak{l}_{2} \rightarrow \mathbb{C}^{\times}$, which by Corollary A.12, defines an isomorphism

$$
b: \operatorname{det}\left(\mathfrak{l}_{1}\right) \otimes \operatorname{det}\left(\mathfrak{l}_{2}\right) \stackrel{\simeq}{\longrightarrow} \mathbb{F}_{p} .
$$

Definition 2.8. We denote by $\theta\left(\tilde{\mathfrak{p}}_{1}, \tilde{\mathfrak{p}}_{2}\right)$ the class of $b\left(\bar{o}_{1} \otimes \bar{o}_{2}\right)$ in $\mathbb{F}_{p}^{\times} /\left(\mathbb{F}_{p}^{\times}\right)^{2}$, and call it the relative orientation of $\tilde{\mathfrak{p}}_{1}$ and $\tilde{\mathfrak{p}}_{2}$.

Remark 2.9. $\theta\left(\tilde{\mathfrak{p}}_{1}, \tilde{\mathfrak{p}}_{2}\right)$ does not depend on the choice of $\bar{o}$ used in its definition. However, as explained in A.4.3, the isomorphism (2.3), and so $\theta$ itself, depends on the choice of $\psi_{0}$ ( $\left.\$ 1.6 .3\right)$; see Remark 1.6.

Remark 2.10. Various notations for relative orientations can be found in the literature (for vector spaces rather than finite groups). To facilitate comparison: our $\theta\left(\tilde{\mathfrak{p}}_{1}, \tilde{\mathfrak{p}}_{2}\right)$ would be called $d_{ \pm}\left(\tilde{\mathfrak{p}}_{2}, \tilde{\mathfrak{p}}_{1}\right)$ in [PPS00], but differs by a sign $(-1)^{m(m-1) / 2}$, with $m:=$ len $\mathfrak{l}_{1}$, from what would be $\operatorname{det}\left(A_{\mathfrak{p}_{2} \mathfrak{p}_{1}}\right)$ in [Per81, LP81] or $\operatorname{det} g_{\tilde{\mathfrak{p}}_{2}, \tilde{\mathfrak{p}}_{1}}$ in LV80. In making these comparisons, it is important to remember our convention $\$ 1.6 .4$.

\subsection{The Heisenberg case.}

2.5.1. Heisenberg Lie rings. Let $(A, \omega)$ be a symplectic module; i.e., $A$ is a finite abelian $p$-group and $\omega$ is a symplectic form (see $₫ \mathbb{B}$.1 for terminology). Let $\mathfrak{z} \subset \mathbb{C}^{\times}$ be the image of $\omega$. The Heisenberg Lie ring associated to $(A, \omega)$ is the abelian group $\mathfrak{h}:=A \oplus \mathfrak{z}$, equipped with the bracket

$$
[(a, s),(b, t)]=(0, \omega(a, b)), \quad \forall a, b \in A, \quad \forall s, t \in \mathfrak{z} .
$$

Note that $[\mathfrak{h}, \mathfrak{h}]=Z(\mathfrak{h})=\mathfrak{z}$, where $\mathbf{Z}(\mathfrak{h})$ denotes the center of $\mathfrak{h}$.

\footnotetext{
${ }^{3}$ Suppose $x \in \mathfrak{p}_{1}$ is such that $B(x, y)=1$ for all $y \in \mathfrak{p}_{2}$. Then the group generated by $x$ and $\mathfrak{p}_{2}$ is an isotropic subgroup of $\mathfrak{g}$. As $\mathfrak{p}_{2}$ is a maximal isotropic subgroup, this implies $x \in \mathfrak{p}_{2}$.
} 
2.5.2. Polarizations. Let $f \in \mathfrak{h}^{*}$ and suppose $\psi: \mathfrak{z} \rightarrow \mathbb{C}^{\times}$is the restriction of $f$ to the center $Z(\mathfrak{h})=\mathfrak{z}$. Note that $\psi \circ \omega$ defines a symplectic pairing on $\bar{A}:=$ $A / \operatorname{Ker}(\psi \circ \omega)$. Let $\pi: A \rightarrow \bar{A}$ be the canonical projection. Then

$$
L \mapsto \pi^{-1}(L) \oplus \mathfrak{z}
$$

defines a bijection between Lagrangians of $\bar{A}$ and polarizations of $f$. In particular, if $\psi \circ \omega$ is nondegenerate, then polarizations for $f$ are in bijection with Lagrangians in $A$.

Remark 2.11. The pairing $\psi \circ \omega$ is nondegenerate if $\psi$ is injective, and in particular when $f$ is the projection $\mathfrak{h} \rightarrow \mathfrak{z} \subset \mathbb{C}^{\times}$.

2.5.3. All polarizations for Heisenberg Lie rings are neighbors. Suppose $\mathfrak{p}_{1}$ and $\mathfrak{p}_{2}$ are polarizations for $f \in \mathfrak{h}^{*}$. Then

$$
\left[\mathfrak{p}_{1}, \mathfrak{p}_{2}\right] \subseteq[\mathfrak{h}, \mathfrak{h}]=\mathfrak{z} \subseteq \mathfrak{p}_{1} \cap \mathfrak{p}_{2} .
$$

2.5.4. Oriented polarizations. Let $\tilde{L}_{1}, \tilde{L}_{2}$ be oriented Lagrangians in $A$. We can define the relative orientation $\theta\left(\tilde{L}_{1}, \tilde{L}_{2}\right)$ by modifying the procedure of $\$ 2.4$ in an obvious way.

Suppose that $\psi \circ \omega$ is nondegenerate. Let $\mathfrak{p}_{i}:=L_{i} \oplus \mathfrak{z}$. According to 22.5 .2 each $\mathfrak{p}_{i}$ is a polarization for $f$. Choosing an orientation $o \in \operatorname{det}(\mathfrak{z})$, we obtain an orientation $o_{i} \in \operatorname{det}\left(\mathfrak{p}_{i}\right)=\operatorname{det}\left(L_{i}\right) \otimes \operatorname{det}(\mathfrak{z})$. It is easy to show that $\theta\left(\tilde{\mathfrak{p}}_{1}, \tilde{\mathfrak{p}}_{2}\right)=\theta\left(\tilde{L}_{1}, \tilde{L}_{2}\right)$.

\section{ThE ORBIT METHOD}

Here we describe in more detail the orbit method of \$1.1, following the approach of [BD06, §2]. The example of the Heisenberg group is worked out in $§ 3.4$

3.1. The Lie ring of a finite $p$-group. Let $\mathfrak{n i l p}_{p}$ denote the category of nilpotent Lie rings of order a power of $p$ and nilpotence class strictly less than $p$. To every $\mathfrak{g} \in \mathfrak{n i l} \mathfrak{p}_{p}$ we associate a finite $p$-group $G=\operatorname{Exp}(\mathfrak{g})$ as follows. $\operatorname{Exp}(\mathfrak{g})$ has the same underlying set as $\mathfrak{g}$ and the group operation is defined by

$$
x * y:=\sum_{i<p} \mathrm{CH}_{i}(x, y) \quad x, y \in \mathfrak{g},
$$

where $\mathrm{CH}_{i}$ is the homogenous component of degree $i$ of the Campbell-Hausdorff series

$$
\begin{aligned}
\mathrm{CH}(x, y) & :=\log (\exp (x) \exp (y)) \\
& =x+y+\frac{1}{2}[x, y]+\text { (higher degree terms). }
\end{aligned}
$$

It is well known that $\mathrm{CH}_{i}$ is a polynomial with coefficients in $\mathbb{Z}\left[\frac{1}{i !}\right]$. Hence the group operation is well defined. It is easy to check that if $\varphi: \mathfrak{g} \rightarrow \mathfrak{g}^{\prime}$ is a morphism of Lie rings, then $\varphi$, viewed as a map $\operatorname{Exp}(\mathfrak{g}) \rightarrow \operatorname{Exp}\left(\mathfrak{g}^{\prime}\right)$, is a group homomorphism. Let $\mathrm{Nilp}_{p}$ denote the category of finite $p$-groups of nilpotence class strictly less than $p$.

Theorem 3.1 ([Laz54]). Exp: $\mathfrak{n i l}_{p} \rightarrow \operatorname{Nilp}_{p}$ is an equivalence of categories.

We will denote the inverse of Exp by $\log$. If $G \in \operatorname{Nilp}_{p}$, we refer to $\log (G)$ as the Lie ring associated to $G$. We denote the identity maps between $\mathfrak{g}$ and $G$ by $\log : G \rightarrow \mathfrak{g}$ and exp: $\mathfrak{g} \rightarrow G$. 
3.2. Coadjoint orbits. Let $G \in \operatorname{Nilp}_{p}$ and let $\mathfrak{g}:=\log (G)$. $G$ acts on $\mathfrak{g}$ by conjugation. This is known as the adjoint action. The corresponding right action of $G$ on $\mathfrak{g}^{*}:=\operatorname{Hom}\left(\mathfrak{g}, \mathbb{C}^{\times}\right)$is known as the coadjoint action. The orbits of the action of $G$ on $\mathfrak{g}^{*}$ are known as the coadjoint orbits.

3.3. The orbit method. Fix $G \in \operatorname{Nilp}_{p}, \mathfrak{g}:=\log (G)$ and $f \in \mathfrak{g}^{*}$ and let $\Omega \subseteq \mathfrak{g}^{*}$ denote the coadjoint orbit containing $f$.

Let $\mathfrak{p}$ be a polarization for $f$ and let $P:=\operatorname{Exp}(\mathfrak{p}) \subseteq G$. Then $\chi_{f}: P \rightarrow \mathbb{C}^{\times}$ defined by

$$
\chi_{f}(x)=f(\log x)
$$

is a one-dimensional representation of $P$. Let $\rho_{f, \mathfrak{p}}:=\operatorname{ind}_{P}^{G} \chi_{f}$. As in $\$ 1.2$, we write $V_{f, \mathfrak{p}}$ for the representation space of $\rho_{f, \mathfrak{p}}$.

Theorem 3.2 ([Kir62], [BD06, §2]). The representation $\rho_{f, \mathfrak{p}}$ is irreducible and its character is given by

$$
g \mapsto \frac{1}{\sqrt{|\Omega|}} \sum_{f \in \Omega} f(\log g), \quad \forall g \in G .
$$

For each coadjoint orbit $\Omega$, choose $f \in \Omega$ and a polarization $\mathfrak{p}$ of $f$. Then the map $\Omega \mapsto \rho_{\Omega}:=\rho_{f, \mathfrak{p}}$ is a bijection between the set of coadjoint orbits and the set of isomorphism classes of irreducible representations of $G$.

Remark 3.3. One can show that the size $|\Omega|$ of each coadjoint orbit is a perfect square (cf. [BD06, §2]), so $\sqrt{|\Omega|}$ is a positive integer. This is the finite analogue of the well-known fact that coadjoint orbits of Lie groups are even dimensional, since they carry symplectic structures; cf. Kir04, \$1.2].

3.4. Example: Heisenberg groups. Suppose $\mathfrak{h}$ is the Heisenberg Lie ring associated to a symplectic module $(A, \omega)$ as in $\$ 2.5$. Then the underlying set of the Heisenberg group $H:=\operatorname{Exp}(\mathfrak{h})$ is $A \times \mathfrak{z}$ and the multiplication is given by

$$
(a, s) *(b, t)=\left(a+b, s \cdot t \cdot \omega(a, b)^{1 / 2}\right)
$$

for all $a, b \in A$ and $s, t \in \mathfrak{z}$. (Note that $\mathfrak{z}$ is written multiplicatively, and $\omega(a, b)$ has a unique square root in $\mathfrak{z}$.)

3.4.1. Coadjoint orbits. The adjoint action $H$ on $\mathfrak{h}$ is easy to compute: given $(a, t) \in$ $H$, we have

$$
\operatorname{Ad}(a, t): \mathfrak{h} \rightarrow \mathfrak{h}, \quad(b, s) \mapsto(b, s \cdot \omega(a, b)) .
$$

The coadjoint action of $H$ on $f \in \mathfrak{h}^{*}$ is given by

$$
\left[\operatorname{Ad}^{*}(a, t) f\right](b, s):=f([\operatorname{Ad}(a, t)](b, s))=f(b, s \cdot \omega(a, b)) .
$$

Let $\psi$ denote the restriction of $f$ to $Z(\mathfrak{h})=\mathfrak{z}$. Then the coadjoint orbit containing $f$ has size $|A / \operatorname{Ker}(\psi \circ \omega)|$.

3.4.2. The Stone-von Neumann Theorem. Theorem 3.2 has the following special case, known traditionally as the Stone-von Neumann Theorem. Choose a nontrivial character $\psi: \mathfrak{z} \rightarrow \mathbb{C}^{\times}$, and define $f \in \mathfrak{h}^{*}$ by $f(v, t)=\psi(t)$. According to 2.5.2, polarizations of $f$ are of the form $\mathfrak{p}:=L \oplus \mathfrak{z}$ where $L$ is a Lagrangian in $A$. The orbit method states that the corresponding representation $\rho_{f, \mathfrak{p}}:=\operatorname{ind}_{P}^{H} \chi_{f}$ is irreducible, and its isomorphism class does not depend on $L$. Moreover, this is the unique isomorphism class of representations with central character $\psi$. 


\section{The REDUCTION PROCESS}

4.1. Reduction of Lie rings to the Heisenberg case. Let $\mathfrak{g}$ be a Lie ring. For every $f \in \mathfrak{g}^{*}$, let $B_{f}: \mathfrak{g} \otimes \mathfrak{g} \rightarrow \mathbb{C}^{\times}$denote the skew pairing

$$
B_{f}(x, y)=f([x, y]), \quad \forall x, y \in \mathfrak{g} .
$$

Let $\omega$ be the induced symplectic form on $\mathfrak{g} / \operatorname{Ker}\left(B_{f}\right)$.

Definition 4.1. We will denote by $\mathfrak{g}^{f}$ the Heisenberg Lie ring associated to the pair $\left(\mathfrak{g} / \operatorname{Ker}\left(B_{f}\right), \omega\right)$ as in $\$ 2.5$ so $\mathfrak{g}^{f}$ equals $\mathfrak{g} / \operatorname{Ker}\left(B_{f}\right) \oplus \mathfrak{z}$ as an abelian group. If $\mathfrak{p} \subseteq \mathfrak{g}$ is a subgroup containing $\operatorname{Ker}\left(B_{f}\right)$, we denote by $\mathfrak{p}^{f} \subseteq \mathfrak{g}^{f}$ the subgroup $\mathfrak{p} / \operatorname{Ker}\left(B_{f}\right) \oplus \mathfrak{z}$. Finally, let $\bar{f} \in\left(\mathfrak{g}^{f}\right)^{*}$ denote the composition

$$
\bar{f}: \mathfrak{g}^{f} \rightarrow \mathfrak{z} \subset \mathbb{C}^{\times} .
$$

4.1.1. Polarizations in the reduction process. If $\mathfrak{p}$ is a polarization for $f$, then $\mathfrak{p}^{f}$ is a polarization for $\bar{f}$. By the discussion of $2.5 .2, \mathfrak{p}^{f}=L \oplus \mathfrak{z}$ for some Lagrangian $L \subset \mathfrak{g}^{f}$.

Remark 4.2. The fact that $\mathfrak{p} / \operatorname{Ker} B_{f}$ is a Lagrangian in $\mathfrak{g} / \operatorname{Ker} B_{f}$ shows that $|\mathfrak{p}|=\sqrt{|\mathfrak{g}|\left|\operatorname{Ker} B_{f}\right|}$ (cf. Remark B.4).

4.1.2. Invariance of relative orientation under the reduction process. Let $\tilde{\mathfrak{p}}_{i}=\left(\mathfrak{p}_{i}, o_{i}\right)$, $i=1,2$, be oriented polarizations for $f$. Choose (once and for all) orientations of $\operatorname{Ker}\left(B_{f}\right)$ and $\mathfrak{z}$. Then the isomorphisms

$$
\begin{aligned}
\operatorname{det}(\mathfrak{p}) & \cong \operatorname{det}\left(\mathfrak{p} / \operatorname{Ker}\left(B_{f}\right)\right) \otimes \operatorname{det}\left(\operatorname{Ker}\left(B_{f}\right)\right), \\
\operatorname{det}\left(\mathfrak{p}^{f}\right) & \cong \operatorname{det}\left(\mathfrak{p} / \operatorname{Ker}\left(B_{f}\right)\right) \otimes \operatorname{det}(\mathfrak{z}),
\end{aligned}
$$

determine orientations $o_{i}^{f}$ of $\mathfrak{p}_{i}^{f}$. Let $\tilde{\mathfrak{p}}_{i}^{f}:=\left(\mathfrak{p}_{i}^{f}, o_{i}^{f}\right)$. It is easy to show that $\theta\left(\tilde{\mathfrak{p}}_{1}^{f}, \tilde{\mathfrak{p}}_{2}^{f}\right)=\theta\left(\tilde{\mathfrak{p}}_{1}, \tilde{\mathfrak{p}}_{2}\right)$, independent of the choices made.

4.2. The cocycle of three polarizations. Let $\mathfrak{g}$ be a Lie ring of nilpotence class strictly less than $p$, and $G:=\operatorname{Exp}(\mathfrak{g})$ the corresponding finite $p$-group, as in 3.1 . Fix $f \in \mathfrak{g}^{*}$. For each polarization $\mathfrak{p}$ of $f$, let $\left(V_{f, \mathfrak{p}}, \rho_{f, \mathfrak{p}}\right)$ denote the corresponding irreducible representation of $G$, as in 33.3 . For each pair $\mathfrak{p}_{1}, \mathfrak{p}_{2}$ of polarizations we have the intertwiner $\Phi_{\mathfrak{p}_{1}, \mathfrak{p}_{2}} \in \operatorname{Hom}_{G}\left(V_{f, \mathfrak{p}_{2}}, V_{f, \mathfrak{p}_{1}}\right)$ defined by (1.3). For each triple $\mathfrak{p}_{1}, \mathfrak{p}_{2}, \mathfrak{p}_{3}$ we have the unitary scalar $\alpha\left(\mathfrak{p}_{1}, \mathfrak{p}_{2}, \mathfrak{p}_{3}\right)$ defined by (1.4). Our goal is to prove Theorem 1.7 i.e., that

$$
\alpha\left(\mathfrak{p}_{1}, \mathfrak{p}_{2}, \mathfrak{p}_{3}\right)=\alpha\left(\mathfrak{p}_{1}^{f}, \mathfrak{p}_{2}^{f}, \mathfrak{p}_{3}^{f}\right) .
$$

We proceed in three steps.

Step 1: Basic formula. First let us give a clean formula for $\alpha\left(\mathfrak{p}_{1}, \mathfrak{p}_{2}, \mathfrak{p}_{3}\right)$. Let

$$
S=S\left(\mathfrak{p}_{3}, \mathfrak{p}_{2}, \mathfrak{p}_{1}\right):=\left\{\left(p_{3}, p_{2}, p_{1}\right) \mid p_{i} \in P_{i}, p_{3} p_{2} p_{1}=1\right\} .
$$

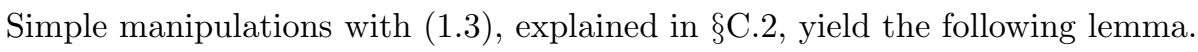

Lemma 4.3. For any polarizations $\mathfrak{p}_{1}, \mathfrak{p}_{2}, \mathfrak{p}_{3}$ of $f$,

$$
\alpha\left(\mathfrak{p}_{1}, \mathfrak{p}_{2}, \mathfrak{p}_{3}\right)=N\left(\mathfrak{p}_{1}, \mathfrak{p}_{2}, \mathfrak{p}_{3}\right) \sum_{\left(p_{3}, p_{2}, p_{1}\right) \in S}\left(\chi_{f}\left(p_{3}\right) \chi_{f}\left(p_{2}\right) \chi_{f}\left(p_{1}\right)\right)^{-1}
$$

where

$$
N\left(\mathfrak{p}_{1}, \mathfrak{p}_{2}, \mathfrak{p}_{3}\right):=\sqrt{\frac{|\mathfrak{g}|\left|\operatorname{Ker} B_{f}\right|}{\left|\mathfrak{p}_{1}\right|\left|\mathfrak{p}_{2}\right|\left|\mathfrak{p}_{3}\right|\left|\mathfrak{p}_{1} \cap \mathfrak{p}_{2}\right|\left|\mathfrak{p}_{2} \cap \mathfrak{p}_{3}\right|\left|\mathfrak{p}_{3} \cap \mathfrak{p}_{1}\right|}}
$$


Step 2: Reduction to neighboring polarizations. Let $m$ be the shortest length of a chain $\mathfrak{p}_{1}=\mathfrak{q}_{1}, \ldots, \mathfrak{q}_{m}=\mathfrak{p}_{2}$ connecting $\mathfrak{p}_{1}$ and $\mathfrak{p}_{2}$ (see Definition 2.5 and Lemma 2.6).

We wish to reduce to the case $m=1$, i.e., the case when $\mathfrak{p}_{1}$ and $\mathfrak{p}_{2}$ are neighbors. First, if $m=0$, the theorem is easy: $\mathfrak{p}_{1}=\mathfrak{p}_{2}, \mathfrak{p}_{1}^{f}=\mathfrak{p}_{2}^{f}$, and, since $\Phi_{\mathfrak{p}_{1}, \mathfrak{p}_{3}}=\Phi_{\mathfrak{p}_{3}, \mathfrak{p}_{1}}^{-1}$, we have

$$
\alpha\left(\mathfrak{p}_{1}, \mathfrak{p}_{2}, \mathfrak{p}_{3}\right)=1=\alpha\left(\mathfrak{p}_{1}^{f}, \mathfrak{p}_{2}^{f}, \mathfrak{p}_{3}^{f}\right) .
$$

Assume now that $m>1$. The identity

$$
\alpha\left(\mathfrak{p}_{1}, \mathfrak{p}_{2}, \mathfrak{p}_{3}\right)=\alpha\left(\mathfrak{p}_{1}, \mathfrak{q}_{2}, \mathfrak{p}_{3}\right) \alpha\left(\mathfrak{q}_{2}, \mathfrak{p}_{2}, \mathfrak{p}_{3}\right) \alpha\left(\mathfrak{p}_{1}, \mathfrak{q}_{2}, \mathfrak{p}_{2}\right)^{-1}
$$

can be deduced by calculating $\Phi_{\mathfrak{p}_{1}, \mathfrak{q}_{2}} \circ \Phi_{\mathfrak{q}_{2}, \mathfrak{p}_{2}} \circ \Phi_{\mathfrak{p}_{2}, \mathfrak{p}_{3}}$ in two different ways:

$$
\begin{aligned}
\left(\Phi_{\mathfrak{p}_{1}, \mathfrak{q}_{2}} \circ \Phi_{\mathfrak{q}_{2}, \mathfrak{p}_{2}}\right) \circ \Phi_{\mathfrak{p}_{2}, \mathfrak{p}_{3}} & =\alpha\left(\mathfrak{p}_{1}, \mathfrak{q}_{2}, \mathfrak{p}_{2}\right) \Phi_{\mathfrak{p}_{1}, \mathfrak{p}_{2}} \circ \Phi_{\mathfrak{p}_{2}, \mathfrak{p}_{3}} \\
& =\alpha\left(\mathfrak{p}_{1}, \mathfrak{q}_{2}, \mathfrak{p}_{2}\right) \alpha\left(\mathfrak{p}_{1}, \mathfrak{p}_{2}, \mathfrak{p}_{3}\right) \Phi_{\mathfrak{p}_{1}, \mathfrak{p}_{3}},
\end{aligned}
$$

while on the other hand,

$$
\begin{aligned}
\Phi_{\mathfrak{p}_{1}, \mathfrak{q}_{2}} \circ\left(\Phi_{\mathfrak{q}_{2}, \mathfrak{p}_{2}} \circ \Phi_{\mathfrak{p}_{2}, \mathfrak{p}_{3}}\right) & =\Phi_{\mathfrak{p}_{1}, \mathfrak{q}_{2}} \circ \alpha\left(\mathfrak{q}_{2}, \mathfrak{p}_{2}, \mathfrak{p}_{3}\right) \Phi_{\mathfrak{q}_{2}, \mathfrak{p}_{3}} \\
& =\alpha\left(\mathfrak{p}_{1}, \mathfrak{q}_{2}, \mathfrak{p}_{3}\right) \alpha\left(\mathfrak{q}_{2}, \mathfrak{p}_{2}, \mathfrak{p}_{3}\right) \Phi_{\mathfrak{p}_{1}, \mathfrak{p}_{3}} .
\end{aligned}
$$

Comparing these expressions yields (4.3).

Now, in each factor on the right of (4.3), the first two polarizations can be joined by a chain of length strictly less than $m$. We can thus reduce by induction to the base case $m=1$.

Step 3: Computation for neighboring polarizations. Suppose that $\mathfrak{p}_{1}$ and $\mathfrak{p}_{2}$ are neighbors. Let

$$
\mathfrak{S}=\left\{\left(p_{3}, p_{2}, p_{1}\right) \mid p_{i} \in \mathfrak{p}_{i}, p_{3}+p_{2}+p_{1}=0\right\}
$$

and let $\mathfrak{R}$ be the equivalence relation on $\mathfrak{S}$ given by

$$
\left(p_{3}, p_{2}, p_{1}\right) \sim\left(a_{13}+p_{3}-a_{32}, a_{32}+p_{2}-a_{21}, a_{21}+p_{1}-a_{13}\right)
$$

for all selections of $\left(a_{32}, a_{21}, a_{13}\right) \in\left(\mathfrak{p}_{3} \cap \mathfrak{p}_{2}\right) \times\left(\mathfrak{p}_{2} \cap \mathfrak{p}_{1}\right) \times\left(\mathfrak{p}_{1} \cap \mathfrak{p}_{3}\right)$.

Lemma 4.4. When $\mathfrak{p}_{1}, \mathfrak{p}_{2}$ are neighbors, and $\mathfrak{p}_{3}$ is arbitrary,

$$
\alpha\left(\mathfrak{p}_{1}, \mathfrak{p}_{2}, \mathfrak{p}_{3}\right)=N^{\prime}\left(\mathfrak{p}_{1}, \mathfrak{p}_{2}, \mathfrak{p}_{3}\right) \sum_{\left(p_{3}, p_{2}, p_{1}\right) \in \mathfrak{S} / \mathfrak{R}} f\left(\frac{1}{2}\left[p_{2}, p_{1}\right]\right)
$$

where

$$
N^{\prime}\left(\mathfrak{p}_{1}, \mathfrak{p}_{2}, \mathfrak{p}_{3}\right):=\sqrt{\frac{|\mathfrak{g}|\left|\mathfrak{p}_{1} \cap \mathfrak{p}_{2}\right|\left|\mathfrak{p}_{2} \cap \mathfrak{p}_{3}\right|\left|\mathfrak{p}_{3} \cap \mathfrak{p}_{1}\right|}{\left|\mathfrak{p}_{1} \cap \mathfrak{p}_{2} \cap \mathfrak{p}_{3}\right|^{2}\left|\mathfrak{p}_{1}\right|\left|\mathfrak{p}_{2}\right|\left|\mathfrak{p}_{3}\right|}} \sqrt{\left|\operatorname{Ker} B_{f}\right|}
$$

Lemma 4.4 follows easily from Lemma 4.3 , The details are given in 9 C.3, Note that the right-hand side of (4.4) remains the same if $\mathfrak{g}, f, \mathfrak{p}_{i}$ are replaced by $\mathfrak{g}^{f}, \bar{f}, \mathfrak{p}_{i}^{f}$. We thus conclude the proof of Theorem 1.7

\section{Compatible intertwiners}

In this section we restate and prove Theorem 1.5. First we calculate the cocycle $\alpha$ of (1.4) in the case of a Heisenberg group. Then we will apply Theorem 1.7 
5.1. The Heisenberg case. Let $(A, \omega)$ be a symplectic module and $\mathfrak{h}$ the corresponding Heisenberg Lie ring, as in $\$ 2.5$. Let $f \in \mathfrak{h}^{*}$ be the projection $\mathfrak{h} \rightarrow \mathfrak{z} \subset \mathbb{C}^{\times}$. By 2.5.2, the map $L \mapsto L \oplus \mathfrak{z}$ is a bijection between Lagrangians in $A$ and polarizations of $f$. Let $\tau\left(L_{1}, L_{2}, L_{3}\right)$ denote the Maslov index of the triple $\left(L_{1}, L_{2}, L_{3}\right)$ (see $\longdiv { \mathrm { B } . 4 }$ ) and let $\gamma$ denote Weil's gamma index (\$B.5).

Proposition 5.1. For any Lagrangians $L_{1}, L_{2}, L_{3}$ in $A$,

$$
\alpha\left(L_{1} \oplus \mathfrak{z}, L_{2} \oplus \mathfrak{z}, L_{3} \oplus \mathfrak{z}\right)=\gamma\left(\tau\left(L_{1}, L_{2}, L_{3}\right)\right) .
$$

The proof is given in $\sqrt{\mathrm{C} .4}$

5.2. The Compatibility Theorem. Fix $\mathfrak{g}, G$, and $f$ as in 44.2 , For any oriented polarizations $\tilde{\mathfrak{p}}_{1}, \tilde{\mathfrak{p}}_{2}$ of $f$, define

$$
\beta\left(\tilde{\mathfrak{p}}_{1}, \tilde{\mathfrak{p}}_{2}\right):=\gamma(1)^{-m\left(\mathfrak{p}_{1}, \mathfrak{p}_{2}\right)^{2}} \gamma\left(\theta\left(\tilde{\mathfrak{p}}_{1}, \tilde{\mathfrak{p}}_{2}\right)\right),
$$

where $m\left(\mathfrak{p}_{1}, \mathfrak{p}_{2}\right):=\operatorname{len}\left(\mathfrak{p}_{1}\right)-\operatorname{len}\left(\mathfrak{p}_{1} \cap \mathfrak{p}_{2}\right)-1$, and $\theta\left(\tilde{\mathfrak{p}}_{1}, \tilde{\mathfrak{p}}_{2}\right)$ is the relative orientation of $\tilde{\mathfrak{p}}_{1}$ and $\tilde{\mathfrak{p}}_{2}$ defined in $\sqrt[2.4]{4}$ Our goal is to prove Theorem 1.5. which amounts to the identity

$$
\alpha\left(\mathfrak{p}_{1}, \mathfrak{p}_{2}, \mathfrak{p}_{3}\right)=\beta\left(\tilde{\mathfrak{p}}_{1}, \tilde{\mathfrak{p}}_{2}\right) \beta\left(\tilde{\mathfrak{p}}_{2}, \tilde{\mathfrak{p}}_{3}\right) \beta\left(\tilde{\mathfrak{p}}_{3}, \tilde{\mathfrak{p}}_{1}\right)
$$

for any triple $\tilde{\mathfrak{p}}_{1}, \tilde{\mathfrak{p}}_{2}, \tilde{\mathfrak{p}}_{3}$ of oriented polarizations of $f$.

5.2.1. Theorem 1.7 states that the left-hand side of (5.2) is unchanged if we replace $\mathfrak{p}_{i}$ by $\mathfrak{p}_{i}^{f}$. On the other hand, by the discussion of 4.1.2, the right-hand side is unchanged if we replace $\tilde{\mathfrak{p}}_{i}$ by $\tilde{\mathfrak{p}}_{i}^{f}$. In view of Proposition 5.1, formula (5.2) is a consequence of the following proposition about the Maslov index.

Proposition 5.2. Suppose $\tilde{L}_{1}, \tilde{L}_{2}, \tilde{L}_{3}$ are oriented Lagrangians in a symplectic module. Then,

$$
\beta\left(\tilde{L}_{1}, \tilde{L}_{2}\right) \beta\left(\tilde{L}_{2}, \tilde{L}_{3}\right) \beta\left(\tilde{L}_{3}, \tilde{L}_{1}\right)=\gamma\left(\tau\left(L_{1}, L_{2}, L_{3}\right)\right) .
$$

The proof is given in $\oint$ C.5.

\section{Appendix A. Determinants of Finite abelian groups}

In this appendix we give two definitions of the determinant of a finite abelian $p$ group. In the case when $A$ is a vector space over $\mathbb{F}_{p}$, its determinant $\operatorname{det} A$ is simply the top exterior power of $A$; in general, $\operatorname{det} A$ is a one-dimensional vector space over $\mathbb{F}_{p}$. It is often convenient to consider $\operatorname{det} A$ as a one-dimensional $\mathbb{Z}$-graded vector space (or 'super line', see 4 A.3) sitting in degree len $A$; we denote this super line by $\operatorname{Det} A$.

First (Definition A.3) we define Det to be the universal additive functor on the category $\mathbf{A} \mathbf{b}_{p}$ of finite abelian $p$-groups. In 1 .1. we recall from Del87 the notions of Picard groupoid and additive functor necessary to make sense of this definition. In $\$$ A.2 we recall Deligne's construction of universal additive functors via $K$-theory. In A.3. Theorem A.9 we identify the target of Det as the category of super lines. Finally, in A.4 we prove that the determinant is compatible with Pontryagin duality.

\footnotetext{
${ }^{4}$ Note that $\gamma(a)$ depends only on the class of $a$ in $\mathbb{F}_{p}^{\times} /\left(\mathbb{F}_{p}^{\times}\right)^{2}$. In particular, the formula for $\beta$ is well defined.
} 
In $\$$ A.5 wive a second, more elementary, but less conceptual treatment, using composition series and the Jordan-Hölder theorem. For some related constructions with exterior powers see [RY02, $\S 2]$.

\section{A.1. Universal additive functors.}

A.1.1. Picard groupoids. A Picard groupoid is a monoidal category in which all objects and morphisms are invertible. A 1-morphism of Picard groupoids (or a Picard functor) is a monoidal functor between the corresponding monoidal categories. A Picard groupoid is commutative if the corresponding monoidal category is symmetric.

Let $\mathcal{P}$ be a Picard groupoid. We denote the set of isomorphism classes of $\mathcal{P}$ by $\pi_{0}(\mathcal{P})$. Let $\pi_{1}(\mathcal{M})$ denote the group of automorphisms of the unit object of $\mathcal{M}$. One can show that $\pi_{1}(\mathcal{M})$ is abelian.

A.1.2. Additive functors. Let $\mathcal{M}$ be an exact category (see Qui73). Let ( $\mathcal{M}$, is) be the groupoid whose objects are the same as $\mathcal{M}$ and whose arrows are isomorphisms in $\mathcal{M}$. Let $\mathcal{P}$ be a Picard groupoid.

Definition A.1 ([Del87], §4.3). An additive functor $\mathcal{M} \rightarrow \mathcal{P}$ is the data of

(a) a functor []$:(\mathcal{M}$, is $) \rightarrow \mathcal{P}$;

(b) for every exact sequence

$$
\Sigma:=\left(0 \rightarrow M^{\prime} \rightarrow M \rightarrow M^{\prime \prime} \rightarrow 0\right)
$$

an isomorphism

$$
\{\Sigma\}:[M] \stackrel{\simeq}{\longrightarrow}\left[M^{\prime}\right]+\left[M^{\prime \prime}\right]
$$

functorial for isomorphisms of exact sequences; and

(c) for every zero object of $\mathcal{M}$, an isomorphism $[0] \stackrel{\simeq}{\longrightarrow} 0$, satisfying the following axioms:

(1) If $\varphi: M \rightarrow N$ is an isomorphism and $\Sigma$ is the exact sequence $0 \rightarrow M \rightarrow N$ (resp. $M \rightarrow N \rightarrow 0$ ), then $[\varphi]$ (resp. $[\varphi]^{-1}$ ) equals the composition

$$
\begin{array}{ll} 
& {[M] \stackrel{\{\Sigma\}}{\longrightarrow}[0]+[N] \stackrel{(c)}{\longrightarrow}[N]} \\
(\text { resp. } & [N] \stackrel{\{\Sigma\}}{\longrightarrow}[M]+[0] \stackrel{(c)}{\longrightarrow}[M]) .
\end{array}
$$

(2) If $0 \subseteq M \subseteq N \subseteq P$ is an admissible filtration in $\mathcal{M}$, the diagram of isomorphisms (coming from (b))

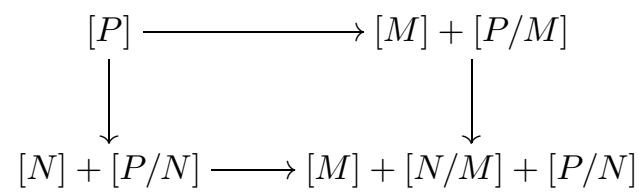

commutes. 
Remark A.2. An additive functor to a commutative Picard groupoid is compatible with the commutativity if for every $M^{\prime}, M^{\prime \prime} \in \mathcal{M}$, the diagram

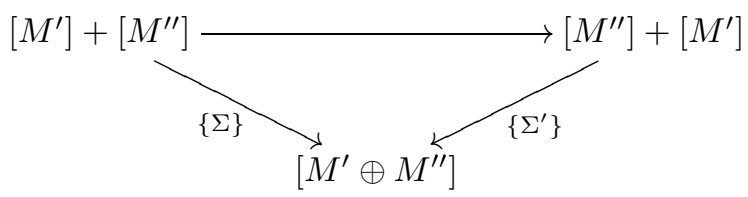

commutes. Here the horizontal arrow is the commutativity isomorphism in $\mathcal{P}$, $\Sigma$ is the exact sequence $M^{\prime} \hookrightarrow M^{\prime} \oplus M^{\prime \prime} \rightarrow M^{\prime \prime}$, and $\Sigma^{\prime}$ is the exact sequence $M^{\prime \prime} \hookrightarrow M^{\prime} \oplus M^{\prime \prime} \rightarrow M^{\prime}$.

A.1.3. Definition of the determinant. Let $\Delta: \mathcal{M} \rightarrow \mathcal{P}$ be an additive functor. We say $\Delta$ is the universal additive functor on $\mathcal{M}$ if for every Picard category $\mathcal{P}^{\prime}$, composition with $\Delta$ defines an equivalence between the groupoid of Picard functors $\mathcal{P}^{\prime} \rightarrow \mathcal{P}$ and the groupoid of additive functors $\mathcal{M} \rightarrow \mathcal{P}$. This characterizes the pair $(\mathcal{P}, \Delta)$ up to an equivalence of Picard categories.

Definition A.3. Let Det be the universal additive functor on the exact category of finite abelian $p$-groups. (The construction of A.2, in particular, Theorem A.5. shows that Det exists.)

In A.3. Theorem A.9, we identify the target of Det with the category of 'super lines' over $\mathbb{F}_{p}$.

\section{A.2. $K$-theoretic construction.}

A.2.1. The $K$-theory space. Let $\mathcal{M}$ be an exact category. To define the algebraic $K$-theory of $\mathcal{M}$, Quillen Qui73 defined a category Q $\mathcal{M}$ which has the same objects as $\mathcal{M}$. (We do not need the precise description of morphisms in this category.) Let $\mathrm{BQ} \mathcal{M}$ denote the geometric realization of $\mathrm{Q} \mathcal{M}$. Direct sums in $\mathcal{M}$ induce operations $\mathrm{Q} \mathcal{M} \times \mathrm{Q} \mathcal{M} \rightarrow \mathrm{Q} \mathcal{M}$ and $\mathrm{BQ} \mathcal{M} \times \mathrm{BQ} \mathcal{M} \rightarrow \mathrm{BQ} \mathcal{M}$ endowing BQ $\mathcal{M}$ with a structure of a commutative $H$-space.

Fix a zero object $0 \in \mathcal{M}$. The $K$-theory space $\mathrm{KM}$ of $\mathcal{M}$ is the based loop space of BQM. One sets $\mathrm{K}_{i}(\mathcal{M}):=\pi_{i}(\mathrm{KM}, 0)$.

A.2.2. The category of exact sequences. Let $\mathcal{E}$ denote the category of exact sequences of the exact category $\mathcal{M}$. For an exact sequence

$$
\Sigma: M^{\prime} \hookrightarrow M \rightarrow M^{\prime \prime}
$$

let $s \Sigma, t \Sigma, q \Sigma$ denote the sub-, total, and quotient objects of $M$. A sequence in $\mathcal{E}$ is called exact if it gives rise to three exact sequences in $\mathcal{M}$ on applying $s, t, q$. The category $\mathcal{E}$ is thus endowed with the structure of an exact category.

Theorem A.4 (Qui73, Theorem 2). The functor

$$
(s, q): \mathrm{Q}(\mathcal{E}) \rightarrow \mathrm{Q}(\mathcal{M}) \times \mathrm{Q}(\mathcal{M})
$$

is a homotopy equivalence. 
A.2.3. The fundamental groupoid of the $K$-theory space. Let $\mathcal{M}$ be an exact category. Let $\mathrm{V}(\mathcal{M})=\Pi(B Q \mathcal{M})$ denote the fundamental groupoid of the $K$-theory space of $\mathcal{M}$ : the objects of $\mathrm{V}(\mathcal{M})$ are loops $\gamma$ in BQM based at 0 . A morphism $\gamma \rightarrow \gamma^{\prime}$ is a homotopy class of based homotopies $\gamma \rightarrow \gamma^{\prime}$. Following Deligne [Del87, we call $\mathrm{V}(\mathcal{M})$ the category of virtual objects of $\mathcal{M}$. The composition of loops defines a multiplication on $\mathrm{V}(\mathcal{M})$ endowing it with a structure of a Picard groupoid. The usual proof that the fundamental group of a group is commutative shows that $\mathrm{V}(\mathcal{M})$ is naturally commutative as a Picard groupoid, being the fundamental groupoid of an $H$-space (\$.2.1).

A.2.4. The universal additive functor. Let $\mathcal{M}$ be an exact category and let $\mathcal{E}$ denote its category of exact sequences. We define an additive functor $\Delta: \mathcal{M} \rightarrow \mathrm{V}(\mathcal{M})$ compatible with the commutativity. Given $M \in \mathcal{M}$, the morphisms $0 \hookrightarrow M$ and $M \rightarrow 0$ define a loop $\Delta(M) \in \mathrm{BQ \mathcal {M }}$ based at 0 , and hence an object of $\mathrm{V}(\mathcal{M})$. Let $M^{\prime} \hookrightarrow M \rightarrow M^{\prime \prime}$ be an exact sequence in $\mathcal{M}$. The homotopy equivalence BQE $\rightarrow$ $\mathrm{BQ} \mathcal{M} \times \mathrm{BQ} \mathcal{M}$ (Theorem A.4) defines an isomorphism $\Delta(M) \rightarrow \Delta\left(M^{\prime}\right)+\Delta\left(M^{\prime \prime}\right)$ in $\mathrm{V}(\mathcal{M})$ making $\Delta$ into an additive functor.

Theorem A.5 ([Del87, $§ 4)$. For any exact category $\mathcal{M}$,

$$
\Delta: \mathcal{M} \rightarrow \mathrm{V}(\mathcal{M})=\Pi(B Q \mathcal{M})
$$

is the universal additive functor.

\section{A.3. The determinant as a super line.}

A.3.1. Super lines. Let $F$ be a field. The commutative Picard groupoid $\mathbf{P i c} \mathbf{c}_{F}$ of super lines on $F$ is defined as follows. The objects of $\mathbf{P i c} \mathbf{c}_{F}$ are pairs $(L, n)$ where $L$ is a one-dimensional vector space over $F$ and $n$ is an integer. A morphism $(L, m) \rightarrow\left(L^{\prime}, m^{\prime}\right)$ exists only if $m=m^{\prime}$, in which case it is any isomorphism $L \rightarrow L^{\prime}$ of vector spaces. The monoidal product is

$$
(L, n) \otimes\left(L^{\prime}, n^{\prime}\right):=\left(L \otimes L^{\prime}, n+n^{\prime}\right) .
$$

The inverse of $(L, n)$ is $\left(L^{\vee},-n\right)$, where $L^{\vee}:=\operatorname{Hom}(L, F)$ is the dual line. Finally, the commutativity constraint $(L, n) \otimes\left(L^{\prime}, n^{\prime}\right) \rightarrow\left(L^{\prime}, n^{\prime}\right) \otimes(L, n)$ is defined using "the Koszul sign rule":

$$
a \otimes a^{\prime} \mapsto(-1)^{n n^{\prime}} a^{\prime} \otimes a, \quad \text { for } a \in L, b \in L^{\prime} .
$$

Remark A.6. Note that $\pi_{0}\left(\mathbf{P i c}_{F}\right)=\mathbb{Z}$ and $\pi_{1}\left(\mathbf{P i c}_{F}\right)=F^{\times}$.

A.3.2. Determinants of vector spaces. Let $\mathbf{V e c t}_{F}$ denote the abelian category of finite dimensional vector spaces over $F$. We define the determinant $\operatorname{Det}_{\mathbf{V e c t}_{F}}$ : Vect $_{F} \rightarrow \mathbf{P i c}_{F}$ so that it assigns to every vector space $V$ the super line $(\operatorname{det}(V), n)$, where $n=\operatorname{dim}(V)$, and $\operatorname{det}(V)=\wedge^{n} V$ denotes the $n^{\text {th }}$ exterior power of $V$. One checks that $\operatorname{Det}_{\text {Vect }_{F}}$ is an additve functor which is compatible with commutativity. This is the reason for choosing the Koszul rule of signs (A.2).

According to Theorem A.5. Det Vect $_{F}$ factors through a Picard functor V( Vect $\left._{F}\right)$ $\rightarrow \mathbf{P i c}_{F}$. The latter functor induces isomorphisms on $\pi_{0}$ and $\pi_{1}$, hence it is an equivalence of Picard groupoids:

Theorem A.7 ([Del87]). Det Vect $_{F}$ is the universal additive functor on the exact category $\operatorname{Vect}_{F}$. 
A.3.3. Determinants of finite abelian p-groups. Let $\mathbf{A b}_{p}$ denote the category of finite abelian $p$-groups. Note that $\operatorname{Vect}_{\mathbb{F}_{p}}$ is the full subcategory of semisimple objects in $\mathbf{A} \mathbf{b}_{p}$.

Theorem A.8 (Qui73, Theorem 4). Suppose $\mathcal{M}$ is an Artinian abelian category. Let $\mathcal{S}$ denote the full subcategory of semisimple objects. The natural functor $\mathrm{Q}(\mathcal{S}) \rightarrow$ $\mathrm{Q}(\mathcal{M})$ is a homotopy equivalence.

By Theorem A.8, the natural functor $\mathrm{QAb}_{p} \rightarrow \mathrm{QVect}_{\mathbb{F}_{p}}$ is a homotopy equivalence. In particular, we have an equivalence of Picard groupoids $\mathrm{V}\left(\mathbf{A} \mathbf{b}_{p}\right) \stackrel{\sim}{\longrightarrow}$ $\mathrm{V}\left(\operatorname{Vect}_{\mathbb{F}_{p}}\right)$. Thus we can rephrase Definition A.3 in the following way.

Theorem A.9. The determinant functor on $\mathbf{A} \mathbf{b}_{p}$ is the composite

$$
\mathbf{A} \mathbf{b}_{p} \stackrel{\Delta}{\longrightarrow} \mathrm{V}\left(\mathbf{A b}_{p}\right) \stackrel{\sim}{\longrightarrow} \mathrm{V}\left(\text { Vect }_{\mathbb{F}_{p}}\right) \stackrel{\sim}{\longrightarrow} \mathbf{P i c}_{\mathbb{F}_{p}}
$$

Definition A.10. We define $\operatorname{det}(A)$ to be the one-dimensional vector space such that $\operatorname{Det}(A)=(\operatorname{det}(A), \operatorname{len}(A))$.

Remark A.11. The fact that $\operatorname{Det}(A)$ has degree $\operatorname{len}(A)$ follows from the analogous statement for vector spaces.

\section{A.4. Compatibility with duality.}

A.4.1. Duality in $\mathbf{P i c}{ }_{F}$. The assignment $(L, n) \mapsto\left(L^{\vee}, n\right)$ defines a Picard functor $\mathfrak{D}_{\mathbf{P i c}_{F}}: \mathbf{P i c}_{F} \rightarrow \mathbf{P i c}_{F}^{o}$, which is an equivalence of Picard categories.

A.4.2. Duality in Vect $_{F}$. The duality functor

$$
\mathfrak{D}_{\operatorname{Vect}_{F}}: \operatorname{Vect}_{F} \rightarrow \operatorname{Vect}_{F}^{o}, \quad V \mapsto V^{\vee}:=\operatorname{Hom}(V, F),
$$

is an equivalence of exact categories. Our convention $\$ 1.6 .4$ defines a natural isomorphism

$$
\operatorname{Det}_{\text {Vect }} \circ \mathfrak{D}_{\mathbf{V e c t}_{F}}=\mathfrak{D}_{\mathbf{P i c}_{F}} \circ \operatorname{Det}_{\mathbf{V e c t}_{F}} .
$$

A.4.3. Duality in $\mathbf{A} \mathbf{b}_{p}$. The Pontryagin duality functor

$$
\mathfrak{D}_{\mathbf{A b}_{p}}: \mathbf{A b}_{p} \rightarrow \mathbf{A b}_{p}^{o} \quad A \mapsto A^{*}:=\operatorname{Hom}\left(A, \mathbb{C}^{\times}\right)
$$

is an equivalence of categories. Let $i$ be the inclusion $\mathbf{V e c t}_{\mathbb{F}_{p}} \hookrightarrow \mathbf{A b}_{p}$. The choice (\$1.6.3) of a nontrivial character $\psi_{0}: \mathbb{F}_{p} \rightarrow \mathbb{C}^{\times}$determines a natural isomorphism $\alpha: \mathfrak{D}_{\mathbf{V e c t}_{\mathrm{F}_{p}}} \rightarrow \mathfrak{D}_{\mathbf{A b}_{p}} \circ i$. This in turn determines a natural isomorphism

$$
\operatorname{Det} \circ \mathfrak{D}_{\mathbf{A b}_{p}}=\mathfrak{D}_{\mathbf{P i}_{\mathbb{i}_{p}}} \circ \text { Det }
$$

as follows:

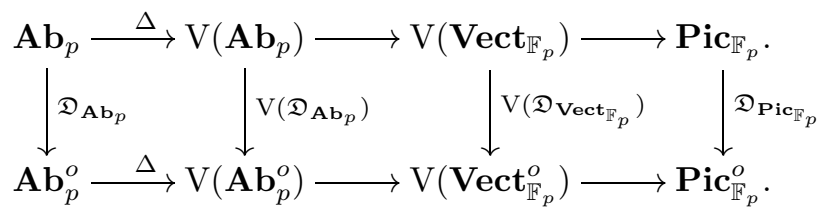

Here the composition along the top and down is $\mathfrak{D}_{\mathbf{P i c}_{\mathbb{F}_{p}}} \circ$ Det, while the composition down and along the bottom is Det $\circ \mathfrak{D}_{\mathbf{A b}_{p}}$. The left-hand square commutes canonically by the functoriality of $\mathrm{V}$; the middle square commutes up to the natural isomorphism $\alpha$; and the right-hand square commutes up to the natural isomorphism (A.3). 
Corollary A.12. Any nondegenerate pairing $\beta: A \otimes B \rightarrow \mathbb{C}^{\times}$determines an isomorphism $\operatorname{det}(\beta): \operatorname{det}(A) \otimes \operatorname{det}(B) \stackrel{\simeq}{\longrightarrow} \mathbb{F}_{p}$.

Remark A.13. As we have defined it, $\operatorname{det}(\beta)$ depends on the choice of $\psi_{0}$. In fact, writing $\mu_{p}$ for the group of $p^{\text {th }}$ roots of unity, one gets an isomorphism $\operatorname{det}(A) \otimes$ $\operatorname{det}(B) \stackrel{\simeq}{\longrightarrow} \mu_{p}^{\otimes \operatorname{len}(A)}$ independent of $\psi_{0}$.

A.5. Elementary description of the determinant. We now give an elementary description of Det, without proofs, as an additive functor ( $\$$ A.1.2 ) from the category $\mathbf{A} \mathbf{b}_{p}$ of finite abelian $p$-groups to the category $\mathbf{P} \mathbf{i c}_{\mathbb{F}_{p}}$ of super lines (A.3.1).

A.5.1. Given a composition series for a finite abelian $p$-group $X$, the associated graded group gr $X$ (i.e., the direct sum of the composition factors) is a vector space over $\mathbb{F}_{p}$. We can then define the determinant Det $X$ to be the determinant of gr $X$ in the sense of vector spaces (see $\$$ A.3.2).

To turn this definition of Det $X$ into an additive functor, we will use a sharp version of the Jordan-Hölder theorem.

A.5.2. To make things precise, we consider a composition series to be a sequence of groups

$$
X=X^{0} \supset X^{1} \supset X^{2} \supset \cdots
$$

such that len $\left(X^{k}\right)=\operatorname{len}(X)-k$. To specify the composition series, it suffices to describe the set $\left\{X^{i}\right\}$ of subgroups. We write $\operatorname{gr}^{m} X:=X^{m} / X^{m+1}$ and $\operatorname{gr} X:=$ $\bigoplus_{m} \operatorname{gr}^{m} X$.

Let $\mathbf{C S}_{p}$ be the category in which the objects are finite abelian $p$-groups equipped with composition series, and the morphisms are arbitrary group homomorphisms. The forgetful functor $\mathbf{C S}_{p} \rightarrow \mathbf{A} \mathbf{b}_{p}$ is an equivalence, and we endow $\mathbf{C} \mathbf{S}_{p}$ with the corresponding structure of an exact category. It suffices to describe the determinant as an additive functor

$$
\operatorname{Det}_{\mathbf{C S}}: \mathbf{C S}_{p} \rightarrow \mathbf{P i c}_{\mathbb{F}_{p}} .
$$

A.5.3. Definition of the functor.

Definition A.14. For $X \in \mathbf{C S}_{p}$, set

$$
\operatorname{Det}_{\mathbf{C S}_{p}} X:=\operatorname{Det}_{\mathbf{V e c t}_{\mathbb{F}_{p}}}(\operatorname{gr} X) \in \mathbf{P i c}_{\mathbb{F}_{p}} \text {. }
$$

Let $\operatorname{det} X$ be the one-dimensional vector space such that $\operatorname{Det}_{\mathbf{C S}_{p}} X=(\operatorname{det} X, \operatorname{len} X)$.

Now suppose, given an isomorphism, $\alpha: X \rightarrow Y$ in $\mathbf{C S}_{p}$. We want to define the corresponding isomorphism

$$
\operatorname{Det}_{\mathbf{C S}_{p}}(\alpha): \operatorname{Det}_{\mathbf{C S}_{p}}(X) \rightarrow \operatorname{Det}_{\mathbf{C S}_{p}}(Y) .
$$

The Jordan-Hölder theorem predicts the existence of an isomorphism $\operatorname{gr}(\alpha): \operatorname{gr} X \rightarrow$ gr $Y$. In fact, as formulated in the next lemma, the proof [Lan05] of Jordan-Hölder determines a canonical map $\operatorname{gr}(\alpha)$, and we can then set

$$
\operatorname{Det}_{\mathbf{C S}_{p}}(\alpha):=\operatorname{Det}_{\operatorname{Vect}_{\mathbb{F}_{p}}}(\operatorname{gr}(\alpha)) .
$$

Lemma A.15 (Jordan-Hölder). Given $\alpha$, there is a unique permutation $\sigma$ of $I:=$ $\{0,1, \ldots$, len $X\}$ with the following property. For each pair $m, n \in I$, define

$$
X^{m} * Y^{n}:=\frac{\alpha\left(X^{m}\right) \cap Y^{n}}{\alpha\left(X^{m+1}\right) \cap Y^{n}+\alpha\left(X^{m}\right) \cap Y^{n+1}} .
$$


Then the natural maps $X^{m} \stackrel{\alpha^{-1}}{\longleftarrow} \alpha\left(X^{m}\right) \cap Y^{\sigma(m)} \longrightarrow Y^{\sigma(m)}$ induce isomorphisms

$$
\operatorname{gr}^{m} X \longleftarrow X^{m} * Y^{\sigma(m)} \longrightarrow \operatorname{gr}^{\sigma(m)} Y .
$$

Definition A.16. Define $\operatorname{Det}_{\mathbf{C S}_{p}}(\alpha)$ by (A.4), where $\operatorname{gr}(\alpha)$ be the isomorphism

$$
\operatorname{gr}(\alpha): \operatorname{gr} X=\bigoplus_{m} \operatorname{gr}^{m} X \rightarrow \bigoplus_{m} \operatorname{gr}^{\sigma(m)} Y=\operatorname{gr} Y
$$

defined on the $m$ th summand by A.5.

Proposition A.17 (Functoriality). For any isomorphisms

$$
\alpha: X \rightarrow Y \quad \text { and } \quad \beta: Y \rightarrow Z
$$

in $\mathbf{C S}_{p}$, we have $\operatorname{Det}_{\mathbf{C S}_{p}}(\beta \circ \alpha)=\operatorname{Det}_{\mathbf{C S}_{p}}(\beta) \circ \operatorname{Det}_{\mathbf{C S}_{p}}(\alpha)$.

Remark A.18. It is not generally true that $\operatorname{gr}(\beta \circ \alpha)=\operatorname{gr}(\beta) \circ \operatorname{gr}(\alpha)$.

\section{A.5.4. Additive structure. Suppose}

$$
\Sigma=\left[0 \rightarrow M^{\prime} \rightarrow M \stackrel{\pi}{\rightarrow} M^{\prime \prime} \rightarrow 0\right]
$$

is a short exact sequence in $\mathbf{C S}_{p}$ (so there are composition series given independently for each of $\left.M, M^{\prime}, M^{\prime \prime}\right)$. We wish to define an isomorphism

$$
\{\Sigma\}: \operatorname{Det}_{\mathbf{C S}_{p}}(M) \rightarrow \operatorname{Det}_{\mathbf{C S}_{p}}\left(M^{\prime}\right) \otimes \operatorname{Det}_{\mathbf{C S}_{p}}\left(M^{\prime \prime}\right)
$$

as in Definition A.1(b).

Let $M_{0}$ be $M$ as a group with the composition series given by the set of subgroups $\left\{M^{\prime, m} \mid 0 \leq m<\operatorname{len} M^{\prime}\right\} \cup\left\{\pi^{-1}\left(M^{\prime \prime}, m\right) \mid 0 \leq m<\right.$ len $\left.M^{\prime \prime}\right\}$. Note that there is a canonical isomorphism

$$
\operatorname{gr} M_{0}=\operatorname{gr} M^{\prime} \oplus \operatorname{gr} M^{\prime \prime} .
$$

Define $\{\Sigma\}$ as the composition

$$
\{\Sigma\}: \operatorname{Det}_{\mathbf{C S}}(M) \rightarrow \operatorname{Det}_{\mathbf{C S}}\left(M_{0}\right) \rightarrow \operatorname{Det}_{\mathbf{C S}_{p}}\left(M^{\prime}\right) \otimes \operatorname{Det}_{\mathbf{C S}_{p}}\left(M^{\prime \prime}\right)
$$

where the first arrow is $\operatorname{Det}_{\mathbf{C S}_{p}}\left(\mathrm{Id}: M \rightarrow M_{0}\right)$ and the second comes from the decomposition (A.6) and the additivity of $\operatorname{Det}_{\mathbf{V e c t}_{\mathbb{F}_{p}}}$.

Finally, as per Definition A.1(c), there is also a canonical isomorphism $z$ : $\operatorname{Det}_{\mathbf{C S}_{p}}(0) \rightarrow \mathbb{F}_{p}$, where 0 is the one-element group with the trivial composition series.

Proposition A.19 (Additivity). The isomorphisms $\{\Sigma\}$ and $z$ make Det $_{\mathbf{C S}_{p}}$ into an additive functor. Moreover, it is a universal additive functor on $\mathbf{C S}_{p}$.

A.5.5. Compatibility with duality. Let $\mathfrak{D C S}_{\mathbf{C S}_{p}}: \mathbf{C S}_{p} \rightarrow \mathbf{C S}_{p}{ }^{\circ}$ be the duality functor: $\mathfrak{D}_{\mathbf{C S}_{p}} X:=\operatorname{Hom}\left(X, \mathbb{C}^{\times}\right)$as a group, with the composition series

$$
\left(\mathfrak{D}_{\mathbf{C S}_{p}} X\right)^{m}:=\operatorname{Hom}\left(X /\left(X^{\operatorname{len}(X)-m}\right), \mathbb{C}^{\times}\right) .
$$

Using the other duality functors of $\$$ A.4, we can define an isomorphism

$$
\operatorname{Det}_{\mathbf{C S}_{p}}\left(\mathfrak{D}_{\mathbf{C S}_{p}}(X)\right) \rightarrow \mathfrak{D}_{\mathbf{P i c}_{\mathbb{F}_{p}}}\left(\operatorname{Det}_{\mathbf{C S}_{p}}(X)\right)
$$


by the following composition:

$$
\begin{gathered}
\operatorname{Det}_{\mathbf{V e c t}_{\mathbb{F}_{p}}}\left(\operatorname{gr}\left(\mathfrak{D}_{\mathbf{C S}_{p}} X\right)\right) \longrightarrow \operatorname{Det}_{\mathbf{V e c t}_{\mathbb{p}_{p}}}\left(\mathfrak{D}_{\mathbf{A b}_{p}}(\operatorname{gr} X)\right) \\
\mathfrak{D}_{\mathbf{P i c}_{\mathbb{F}_{p}}}\left(\operatorname{Det}_{\mathbf{V e c t}_{\mathbb{F}_{p}}}(\operatorname{gr} X)\right) \longleftarrow \operatorname{Det}_{\mathbf{V e c t}_{\mathbb{F}_{p}}}\left(\mathfrak{D}_{\mathbf{V e c t}_{\mathbb{F}_{p}}}^{\downarrow}(\operatorname{gr} X)\right)
\end{gathered}
$$

The top arrow comes from the canonical isomorphism $\operatorname{gr}\left(\mathfrak{D}_{\mathbf{C S}_{p}} X\right)=\mathfrak{D}_{\mathbf{A b}}(\operatorname{gr} X)$; the right arrow is given by the natural transformation $\alpha$ of $\mathrm{A} .4 .3$, depending on $\psi_{0}$; the bottom arrow by the natural transformation (A.3).

Proposition A.20 (Duality). The isomorphisms (A.7) define a natural isomorphism

$$
\operatorname{Det}_{\mathbf{C S}_{p}} \circ \mathfrak{D}_{\mathbf{C S}_{p}}=\mathfrak{D}_{\mathbf{P i}_{\mathbb{F}_{p}}} \circ \operatorname{Det}_{\mathbf{C S}} \text {. }
$$

Corollary A.12 also follows.

\section{Appendix B. Witt group, Maslov index, Gamma index}

In this appendix we develop the Maslov index and some related notions in the setting of finite abelian $p$-groups. Throughout, $p$ is a fixed odd prime.

In $\$$.1 we set out some general conventions, leading up to the study of finite abelian $p$-groups equipped with nondegenerate symmetric forms; we call these quadratic modules. In $\$ \mathrm{~B} .2$ we define the discriminant of a quadratic module as an element of $\mathbb{F}_{p}^{\times} /\left(\mathbb{F}_{p}^{\times}\right)^{2}$. In $\sqrt{\mathrm{B} .3}$ we define the Witt group $\mathbb{W}_{p}$ of quadratic modules. The analogue for symmetric forms on vector spaces is very well known, cf. [Lam05; a general account of Witt groups of symmetric forms on torsion modules can be found in the appendix of BLLV74].

In $\$$ B.4 we define the Maslov index of a collection of Lagrangian subspaces of a symplectic module. Again, the analogue for vector spaces is well known; we follow the development in Tho06. The crucial feature of the present setting is that the Maslov index can be represented as a coboundary (B.4.1).

Finally, in $\$$ B.5. we define a character $\gamma$ of $\mathbb{W}_{p}$, following [Wei64].

B.1. Symplectic and quadratic modules. Let $A$ be a finite abelian $p$-group.

Definition B.1. A pairing $\omega: A \otimes A \rightarrow \mathbb{C}^{\times}$is called skew if $\omega(a, a)=0$ for all $a \in A$, and symmetric if $\omega(a, b)=\omega(b, a)$ for all $a, b \in A$.

Assume from now on that $\omega$ is either skew or symmetric. If $L \subset A$ is a subgroup, write $L^{\perp}$ for the set

$$
L^{\perp}:=\{a \in A \mid \omega(x, a)=1 \forall x \in L\} .
$$

Definition B.2. A subgroup $L \subset A$ is isotropic if $L \subset L^{\perp}$, coisotropic if $L^{\perp} \subset L$, and Lagrangian if $L=L^{\perp}$.

Definition B.3. The kernel $\operatorname{Ker}(\omega) \subset A$ is defined by $\operatorname{Ker}(\omega):=A^{\perp}$. A skew or symmetric pairing is called nondegenerate if $\operatorname{Ker}(\omega)=0$.

Remark B.4. Any skew or symmetric $\omega$ induces a nondegenerate pairing of the same type on $A / \operatorname{Ker}(\omega)$. If $L \subset A$ is Lagrangian and $\omega$ is nondegenerate, then $|A|=|L|^{2}$. 
Definition B.5. A skew pairing is called symplectic if it is nondegenerate. We call a pair $(A, \omega)$ consisting of a finite abelian $p$-group $A$ and a symplectic (resp. nondegenerate symmetric) pairing $\omega$ a symplectic module (resp. quadratic module).

B.2. The discriminant of a quadratic module. Let $(A, q)$ be a quadratic module. According to Corollary A.12, $q$ defines an isomorphism $\operatorname{det}(q): \operatorname{det}(A) \otimes$ $\operatorname{det}(A) \stackrel{\simeq}{\longrightarrow} \mathbb{F}_{p}$. If we choose a trivialization $\operatorname{det}(A) \stackrel{\simeq}{\longrightarrow} \mathbb{F}_{p}, \operatorname{then} \operatorname{det}(q)$ amounts to an isomorphism $\mathbb{F}_{p} \rightarrow \mathbb{F}_{p}$, i.e., to an element $\delta(q) \in \mathbb{F}_{p}^{\times}$. It is easy to show that the class of $\delta(q) \in \mathbb{F}_{p}^{\times} /\left(\mathbb{F}_{p}^{\times}\right)^{2}$ is independent of the chosen trivialization.

Definition B.6. We call $\delta(q) \in \mathbb{F}_{p}^{\times} /\left(\mathbb{F}_{p}^{\times}\right)^{2}$ the discriminant of $(A, q)$.

Remark B.7. When $A$ is a vector space over $\mathbb{F}_{p}$, this discriminant is often called the 'signed discriminant' in the literature (e.g., in [Lam05], [PPS00]). It differs from the traditional discriminant of a quadratic form by a factor of $(-1)^{d(d-1) / 2}$, where $d=$ len $A$, because of our convention $\$ 1.6 .4$

Example B.8. For $a \in \mathbb{F}_{p}^{\times}$, let

$$
\langle a\rangle: \mathbb{F}_{p} \otimes \mathbb{F}_{p} \rightarrow \mathbb{C}^{\times}
$$

be the symmetric form $(x, y) \mapsto \psi_{0}(a x y)$, with $\psi_{0}$ fixed in 91.6 .3 . Note that the isomorphism class of $\langle a\rangle$ depends only on the image of $a$ in $\mathbb{F}_{p}^{\times} /\left(\mathbb{F}_{p}^{\times}\right)^{2}$. Set

$$
\left\langle a_{1}, \ldots, a_{d}\right\rangle:=\left\langle a_{1}\right\rangle \oplus \ldots \oplus\left\langle a_{d}\right\rangle
$$

(orthogonal direct sum). Then

$$
\delta\left(\left\langle a_{1}, \ldots, a_{d}\right\rangle\right)=(-1)^{d(d-1) / 2} a_{1} a_{2} \ldots a_{d} .
$$

B.3. The Witt group. Given any quadratic module $(A, q)$, write $A^{\circ}$ for the same abelian group $A$ equipped with the pairing $q^{\circ}(a, b):=q(a, b)^{-1}$.

Definition B.9. A Lagrangian correspondence $L:\left(A_{1}, q_{1}\right) \rightarrow\left(A_{2}, q_{2}\right)$ is a Lagrangian subgroup of the orthogonal direct sum $A_{1}^{\circ} \oplus A_{2}$. Quadratic modules $\left(A_{1}, q_{1}\right)$ and $\left(A_{2}, q_{2}\right)$ are Witt-equivalent if there exists a Lagrangian correspondence between them.

Example B.10. If $\phi: A \rightarrow A^{\prime}$ is an isomorphism of quadratic modules, then the graph of $\phi$ in $A^{\circ} \oplus A^{\prime}$ is a Lagrangian correspondence. Thus isomorphic quadratic modules are Witt-equivalent.

Two Lagrangian correspondences $L_{21} \subset A_{1}^{\circ} \oplus A_{2}$ and $L_{32} \subset A_{2}^{\circ} \oplus A_{3}$ can be composed to form a Lagrangian correspondence $L_{31} \subset A_{1}^{\circ} \oplus A_{3}$ :

$$
L_{31}:=\left\{(a, c) \mid \exists b \in A_{2} \text { with }(a, b) \in L_{21} \text { and }(b, c) \in L_{31}\right\} .
$$

This defines a category whose objects are quadratic modules and whose morphisms are Lagrangian correspondences. In particular, we obtain

Proposition B.11. Witt-equivalence is an equivalence relation.

Definition B.12. Let $\mathbb{W}_{p}$ denote the set of Witt-equivalence classes of quadratic modules. (Recall that for us a quadratic module is a $p$-group, with $p$ a fixed odd prime.) The orthogonal direct sum operation makes $\mathbb{W}_{p}$ into a monoid, and in fact a group, called the Witt group.

Remark B.13. To see that $\mathbb{W}_{p}$ is a group, note that the inverse of $A$ is $A^{\circ}$. Indeed, the diagonal subgroup of $A^{\circ} \oplus A$ is a Lagrangian correspondence between $A^{\circ} \oplus A$ and the trivial quadratic module. 
B.3.1. Description of the Witt group. Let $\mathbb{W}_{p}^{\prime}$ be the group whose underlying set is $\mathbb{W}_{p}^{\prime}=\mathbb{Z} / 2 \mathbb{Z} \times \mathbb{F}_{p}^{\times} /\left(\mathbb{F}_{p}^{\times}\right)^{2}$ and whose multiplication is given by

$$
(e, d) \cdot\left(e^{\prime}, d^{\prime}\right):=\left(e+e^{\prime},(-1)^{e e^{\prime}} d d^{\prime}\right) .
$$

( $\mathbb{W}_{p}^{\prime}$ is isomorphic to $\mathbb{Z} / 2 \mathbb{Z} \times \mathbb{Z} / 2 \mathbb{Z}$ if -1 is a square in $\mathbb{F}_{p}^{\times}$, and to $\mathbb{Z} / 4 \mathbb{Z}$ otherwise.)

Proposition B.14. The map (len, $\delta): \mathbb{W}_{p} \rightarrow \mathbb{W}_{p}^{\prime}$ is an isomorphism of groups 5 Its inverse $\mathrm{qm}$ is given by

$$
\mathrm{qm}(1, a)=\langle a\rangle \quad \mathrm{qm}(0, a)=\langle-1, a\rangle
$$

in the notation of Example B.8.

The proof in 8 C.6 reduces this proposition to the case of quadratic forms over finite fields studied, for example, in [Lam05, §II.2].

Remark B.15. It follows that any quadratic module $(A, q)$ is Witt-equivalent to $\langle\delta(q)\rangle$ if $\operatorname{len}(A)$ is odd, and to $\langle-1, \delta(q)\rangle$ if $\operatorname{len}(A)$ is even.

B.4. The Maslov index. Let $(A, \omega)$ be a symplectic module. Let $L=\left(L_{1}, \ldots, L_{m}\right)$ be a sequence of Lagrangians in $A$. For notational convenience, set $L_{m+1}:=L_{1}$. Consider the complex of abelian groups

$$
C_{L}:=\left[\bigoplus_{i=1}^{m} L_{i} \cap L_{i+1} \stackrel{\partial}{\longrightarrow} \bigoplus_{i=1}^{m} L_{i} \stackrel{\sum}{\longrightarrow} A\right]
$$

where $\Sigma$ is the summation, and $\partial$ maps $L_{i} \cap L_{i+1}$ into $L_{i} \oplus L_{i+1}$ by $a \mapsto(a,-a)$. Let $T_{L}$ be the homology of $C_{L}$ at the center term. Define a pairing $q_{L}: T_{L} \otimes T_{L} \rightarrow \mathbb{C}^{\times}$ by

$$
q_{L}(a, b):=\prod_{m \geq i>j \geq 1} \omega\left(a_{i}, b_{j}\right) .
$$

Proposition B.16 (cf. [Tho06], Props. 2 and 3). The pairing $q_{L}$ is well-defined, symmetric, and nondegenerate.

Definition B.17. The Maslov index of $L$ is the class of the quadratic module $\left(T_{L}, q_{L}\right)$ in $\mathbb{W}_{p}$.

Proposition B.18 (cf. [Tho08, Prop. 4.1).

(1) The length of $T_{L}$ equals

$$
\frac{m-2}{2} \operatorname{len}(A)-\sum_{1 \leq i \leq m} \operatorname{len}\left(L_{i} \cap L_{i+1}\right)+2 \operatorname{len}\left(\cap_{i=1}^{m} L_{i}\right) .
$$

(2) Equip the $L_{i}$ with arbitrary orientations and let $\tilde{L}_{i}$ denote the resulting oriented Lagrangians (see Definition 2.7). Then

$$
\delta\left(q_{L}\right)=(-1)^{\frac{1}{2} \sum_{i \neq j} m_{i} m_{j}} \prod_{i=1}^{m} \theta\left(\tilde{L}_{i}, \tilde{L}_{i+1}\right)
$$

where $m_{i}:=\operatorname{len}\left(L_{i} / L_{i} \cap L_{i+1}\right)$.

\footnotetext{
${ }^{5}$ It is easy to see that len: $\mathbb{W}_{p} \rightarrow \mathbb{Z} / 2 \mathbb{Z}$ and $\delta: \mathbb{W}_{p} \rightarrow \mathbb{F}_{p}^{\times} /\left(\mathbb{F}_{p}^{\times}\right)^{2}$ are well-defined functions.
} 
Theorem B.19 (cf. [Tho06], Prop. 6). If $L_{1}, \ldots, L_{m}$ are Lagrangians in $(A, \omega)$, then

$$
\tau\left(L_{1}, \ldots, L_{m}\right)=\tau\left(L_{2}, \ldots, L_{m}, L_{1}\right)=-\tau\left(L_{m}, L_{m-1}, \ldots, L_{1}\right)
$$

and

$$
\tau\left(L_{1}, \ldots, L_{m}\right)=\tau\left(L_{1}, \ldots, L_{k}\right)+\tau\left(L_{1}, L_{k}, \ldots, L_{m}\right)
$$

for any $k<m$.

B.4.1. The Maslov cocycle is a coboundary. Let $(A, \omega)$ be a symplectic module. Let $\tilde{L}_{1}, \tilde{L}_{2}$ be oriented Lagrangians in $A$. Set

$$
\Theta\left(\tilde{L}_{1}, \tilde{L}_{2}\right)=\operatorname{qm}\left(\operatorname{len}\left(L_{1} / L_{1} \cap L_{2}\right), \theta\left(\tilde{L}_{1}, \tilde{L}_{2}\right)\right) \in \mathbb{W}_{p},
$$

where $\theta$ is the relative orientation (\$2.5.4), and qm was defined in Proposition B.14 The following result is a restatement of [PPS00, Prop. 2.1, in the finite group setting; it follows easily from (B.1) and Proposition B.18

Proposition B.20. Let $\tilde{L}_{i}=\left(L_{i}, u_{i}\right), 1 \leq i \leq m$ be oriented Lagrangians. Then we have

$$
\tau\left(L_{1}, \ldots, L_{m}\right)=\sum_{i \in \mathbb{Z} / m \mathbb{Z}} \Theta\left(\tilde{L}_{i}, \tilde{L}_{i+1}\right) .
$$

\section{B.5. The $\gamma$-index (after A. Weil Wei64).}

Definition B.21. For any quadratic module $(A, q)$, define

$$
\gamma(q)=\frac{1}{\sqrt{|A|}} \sum_{a \in A} q(a, a / 2)
$$

For any $a \in \mathbb{F}_{p}^{\times}$, write $\gamma(a)$ for $\gamma(\langle a\rangle)$ (in the notation of Example B.8).

Remark B.22. In (1.6) we chose $\psi_{0}(x)=\exp (2 \pi i x / p)$ for concreteness.

Proposition B.23. This $\gamma$ defines a homomorphism $\gamma: \mathbb{W}_{p} \rightarrow \mathbb{C}^{\times}$.

The proof is given in $₫ \mathbb{C}$.7. Since $\left|\mathbb{W}_{p}\right|=4$ by Proposition B.14, we obtain:

Corollary B.24. For any quadratic module $(A, q), \gamma(q)$ is a fourth root of unity.

Combining Remark B.15 and Proposition B.23 we obtain:

Corollary B.25. Let $(A, q)$ be a quadratic module. Ther 6

$$
\gamma(q)=\gamma(1)^{-(d-1)^{2}} \gamma(\delta(q)) .
$$

Finally, one has the following useful result.

Corollary B.26. For any $a, b \in \mathbb{F}_{p}^{\times}, \gamma(a) \gamma(b)=\gamma(1) \gamma(a b)$.

This follows from the equality $\langle a, b\rangle=\langle 1, a b\rangle$ in $\mathbb{W}_{p}$, using the notation of Example B.8.

Remark B.27 (Explicit values). Recall that the definition of $\langle a\rangle$ in Example B.8 depends on the choice of the additive character $\psi_{0}$. For some $m$ coprime to $p$ we have $\psi_{0}(x)=e^{2 \pi i m x / p}$, and one can show that $\gamma(a)=\left(\frac{a m}{p}\right) \epsilon_{p}$, where $\left(\frac{a m}{p}\right)$ is the Legendre symbol and

$$
\epsilon_{p}=\left\{\begin{array}{lll}
-1 & p \equiv 1 & \bmod 4 \\
-i & p \equiv 3 & \bmod 4
\end{array}\right.
$$

\footnotetext{
${ }^{6}$ Note that $(d-1)^{2} \equiv 0 \bmod 4$ if $d$ is odd, and $(d-1)^{2} \equiv 1 \bmod 4$ if $d$ is even.
} 


\section{Appendix C. Proofs}

C.1. Lemma 2.6. We proceed by induction on the cardinality of $\mathfrak{g}$. Set $\mathfrak{g}_{0}:=$ $Z(\mathfrak{g}) \cap \operatorname{ker}(f)$. Then $\mathfrak{g}_{0}$ is an ideal of $\mathfrak{g}$ contained in every polarization of $f$. Moreover, $f$ descends to a character of the Lie ring $\mathfrak{g} / \mathfrak{g}_{0}$, for which $\mathfrak{p}_{1} / \mathfrak{g}_{0}$ and $\mathfrak{p}_{2} / \mathfrak{g}_{0}$ are polarizations. If $\mathfrak{g}_{0} \neq 0$, then by induction we obtain a chain in $\mathfrak{g} / \mathfrak{g}_{0}$, and it lifts to a chain in $\mathfrak{g}$. We can now, therefore, assume that $\mathfrak{g}_{0}=0$.

If $\mathfrak{g}$ is abelian, then $\mathfrak{g}$ itself is the unique polarization of $f$, and there is nothing to prove. Assuming, then, that $\mathfrak{g}$ is not abelian, we can choose $x \in \mathfrak{g}$ such that $0 \neq[\mathfrak{g}, x] \subset Z(\mathfrak{g})$. Using the skew form $(a, b) \mapsto f([a, b])$ on $\mathfrak{g}$ (see $\oint \mathrm{B} .1)$, let $\mathfrak{h}=\{x\}^{\perp}$. Then $\mathfrak{h}$ is a proper coisotropic ideal in $\mathfrak{g}$.

Define $\mathfrak{p}_{i}^{\prime}=\mathfrak{p}_{i} \cap \mathfrak{h}+\mathfrak{h}^{\perp}$. It is easy to check that $\mathfrak{p}_{i}^{\prime}$ is a polarization and that it is a neighbor of $\mathfrak{p}_{i}$. It follows that $\mathfrak{p}_{1}^{\prime}$ and $\mathfrak{p}_{2}^{\prime}$ are polarizations for the restriction of $f$ to $\mathfrak{h}$, and, since $\mathfrak{h}$ is strictly smaller than $\mathfrak{g}$, they are joined by a chain in $\mathfrak{h}$, so $a$ fortiori in $\mathfrak{g}$. In total, we have constructed a chain $\mathfrak{p}_{1}, \mathfrak{p}_{1}^{\prime}, \ldots, \mathfrak{p}_{2}^{\prime}, \mathfrak{p}_{2}$ as desired.

C.2. Lemma 4.3. Let $\delta \in V_{f, p_{1}}$ be supported (as a function on $G$ ) on $P_{1}$ and equal there to $\delta(g)=\chi_{f}(g)$. We have

$$
\begin{aligned}
\alpha\left(\mathfrak{p}_{1}, \mathfrak{p}_{2}, \mathfrak{p}_{3}\right) & =\Phi_{\mathfrak{p}_{1}, \mathfrak{p}_{2}} \circ \Phi_{\mathfrak{p}_{2}, \mathfrak{p}_{3}} \circ \Phi_{\mathfrak{p}_{3}, \mathfrak{p}_{1}} \delta(1) \\
& =N_{0}\left(\mathfrak{p}_{1}, \mathfrak{p}_{2}, \mathfrak{p}_{3}\right) \sum_{p_{3}, p_{2}, p_{1}}\left(\chi_{f}\left(p_{3}\right) \chi_{f}\left(p_{2}\right) \chi_{f}\left(p_{1}\right)\right)^{-1} \delta\left(p_{3} p_{2} p_{1}\right)
\end{aligned}
$$

where $N_{0}\left(\mathfrak{p}_{1}, \mathfrak{p}_{2}, \mathfrak{p}_{3}\right):=\left(\left|\mathfrak{p}_{1}\right|\left|\mathfrak{p}_{2}\right|\left|\mathfrak{p}_{3}\right|\left|\mathfrak{p}_{1} \cap \mathfrak{p}_{2}\right|\left|\mathfrak{p}_{2} \cap \mathfrak{p}_{3} \| \mathfrak{p}_{3} \cap \mathfrak{p}_{1}\right|\right)^{-1 / 2}$. The sums are over all $p_{i} \in P_{i}$, but the summand is only nonzero when $p_{1}^{\prime}:=\left(p_{3} p_{2}\right)^{-1}$ lies in $P_{1}$. Writing $\delta\left(p_{3} p_{2} p_{1}\right)=\chi_{f}\left(p^{\prime}\right)^{-1} \chi_{f}\left(p_{1}\right)$ we obtain simply

$$
\alpha\left(\mathfrak{p}_{1}, \mathfrak{p}_{2}, \mathfrak{p}_{3}\right)=N_{0}\left(\mathfrak{p}_{1}, \mathfrak{p}_{2}, \mathfrak{p}_{3}\right) \sum_{p_{1} \in P_{1},\left(p_{3}, p_{2}, p_{1}^{\prime}\right) \in S}\left(\chi_{f}\left(p_{3}\right) \chi_{f}\left(p_{2}\right) \chi_{f}\left(p_{1}^{\prime}\right)\right)^{-1}
$$

The summand is independent of $p_{1}$, thus introducing a factor of $\left|\mathfrak{p}_{1}\right|=\sqrt{|\mathfrak{g}|\left|\operatorname{Ker} B_{f}\right|}$ (cf. Remark 4.2) to yield the desired formula (4.2).

C.3. Lemma 4.4, In (4.2) we have $p_{3}=\left(p_{2} p_{1}\right)^{-1}$. Looking at the CampbellHausdorff series, the fact that $\mathfrak{p}_{1}$ and $\mathfrak{p}_{2}$ are neighboring polarizations implies that

$$
-\log p_{3} \equiv \log p_{1}+\log p_{2}+\frac{1}{2}\left[\log p_{2}, \log p_{1}\right] \quad \bmod \mathfrak{p}_{1} \cap \mathfrak{p}_{2} \cap \operatorname{ker} f .
$$

In particular, $-\log p_{3}-\log p_{1}$ lies in $\mathfrak{p}_{2}$, and

$$
\left(p_{3}, p_{2}, p_{1}\right) \mapsto\left(q_{3}, q_{2}, q_{1}\right):=\left(\log p_{3},-\log p_{3}-\log p_{1}, \log p_{1}\right)
$$

is a well-defined bijection $S \rightarrow \mathfrak{S}$. We use this bijection to rewrite (4.2) as a sum over S. Using (C.1) we calculate that the summand

$$
\left(\chi_{f}\left(p_{3}\right) \chi_{f}\left(p_{2}\right) \chi_{f}\left(p_{1}\right)\right)^{-1}=f\left(\frac{1}{2}\left[\log p_{2}, \log p_{1}\right]\right)=f\left(\frac{1}{2}\left[q_{2}, q_{1}\right]\right),
$$

so we obtain

$$
\alpha\left(\mathfrak{p}_{1}, \mathfrak{p}_{2}, \mathfrak{p}_{3}\right)=N\left(\mathfrak{p}_{1}, \mathfrak{p}_{2}, \mathfrak{p}_{3}\right) \sum_{\left(q_{3}, q_{2}, q_{1}\right) \in \mathfrak{S}} f\left(\frac{1}{2}\left[q_{2}, q_{1}\right]\right) .
$$

Now it is easy to verify that the summand depends only on the class of $\left(q_{3}, q_{2}, q_{1}\right)$ in $\mathfrak{S} / \mathfrak{R}$. That class has size

$$
\frac{\left|\mathfrak{p}_{1} \cap \mathfrak{p}_{2}\right|\left|\mathfrak{p}_{2} \cap \mathfrak{p}_{3}\right|\left|\mathfrak{p}_{3} \cap \mathfrak{p}_{1}\right|}{\left|\mathfrak{p}_{1} \cap \mathfrak{p}_{2} \cap \mathfrak{p}_{3}\right|}=\frac{N^{\prime}\left(\mathfrak{p}_{1}, \mathfrak{p}_{2}, \mathfrak{p}_{3}\right)}{N\left(\mathfrak{p}_{1}, \mathfrak{p}_{2}, \mathfrak{p}_{2}\right)},
$$

so we obtain (4.4). 
C.4. Proposition 5.1. Since all polarizations in a Heisenberg Lie ring are neighbors (2.5.3), we may use Lemma 4.4, The order-reversing map

$$
F: L_{1} \oplus L_{2} \oplus L_{3} \rightarrow \mathfrak{p}_{3} \oplus \mathfrak{p}_{2} \oplus \mathfrak{p}_{1}
$$

defines a linear isomorphism from $T_{L}\left(\$ \overline{B .4}\right.$, with $\left.L:=\left(L_{1}, L_{2}, L_{3}\right)\right)$ to $\mathfrak{S} / \mathfrak{R}$. Using this to rewrite (4.4) as a sum over $T_{L}$, we find

$$
\alpha\left(\mathfrak{p}_{1}, \mathfrak{p}_{2}, \mathfrak{p}_{3}\right)=N^{\prime}\left(\mathfrak{p}_{1}, \mathfrak{p}_{2}, \mathfrak{p}_{3}\right) \sum_{a \in T_{L}} q_{L}(a, a / 2) .
$$

It only remains to compare this with the definition (B.4) of $\gamma$.

C.5. Proposition 5.2, By Propositions B.20 and B.23, we have

$$
\gamma\left(\tau\left(L_{1}, L_{2}, L_{3}\right)\right)=\gamma\left(\Theta\left(\tilde{L}_{1}, \tilde{L}_{2}\right)\right) \gamma\left(\Theta\left(\tilde{L}_{2}, \tilde{L}_{3}\right)\right) \gamma\left(\Theta\left(\tilde{L}_{3}, \tilde{L}_{1}\right)\right) .
$$

Therefore, it remains to check that

$$
\gamma\left(\Theta\left(\tilde{L}_{1}, \tilde{L}_{2}\right)\right)=\beta\left(\tilde{L}_{1}, \tilde{L}_{2}\right):=\gamma(1)^{-m\left(L_{1}, L_{2}\right)^{2}} \gamma\left(\theta\left(\tilde{L}_{1}, \tilde{L}_{2}\right)\right) .
$$

But this follows immediately from Corollary B.25 and the definition (B.3) of $\Theta$.

C.6. Proposition B.14, The map (len, $\delta$ ) is surjective, since qm is a right inverse. So it will suffice to show that $\mathbb{W}_{p}$ has four elements.

Suppose $(A, q)$ is a quadratic module, and $n$ is the smallest integer such that $p^{n} A=0$. Then $A_{n-1}:=p^{n-1} A$ is an isotropic subgroup of $A$, and $A$ is Wittequivalent to $A_{n-1}^{\perp} / A_{n-1}$ :

$$
\left\{(a, b) \in A^{\circ} \oplus A_{n-1}^{\perp} / A_{n-1} \mid a \equiv b \bmod A_{n-1}\right\}
$$

is a Lagrangian correspondence. Iterating this process, we see that every quadratic module is Witt-equivalent to a $p$-torsion one, i.e., to a vector space over $\mathbb{F}_{p}$. A symmetric form on a vector space can be diagonalized; this shows that $\mathbb{W}_{p}$ is generated by $\langle 1\rangle$ and $\langle a\rangle$ where $a \in \mathbb{F}_{p}^{\times}$is not a square.

If -1 is a square in $\mathbb{F}_{p}^{\times}$, then $\langle 1\rangle=\langle-1\rangle=-\langle 1\rangle$ in $\mathbb{W}_{p}$, so that $\langle 1,1\rangle=0$ (and, similarly, $\langle a, a\rangle=0)$ in $\mathbb{W}_{p}$; thus $\mathbb{W}_{p}$ is isomorphic to $\mathbb{Z} / 2 \mathbb{Z} \times \mathbb{Z} / 2 \mathbb{Z}$.

If -1 is not a square, then $\langle a\rangle=\langle-1\rangle=-\langle 1\rangle$ in $\mathbb{W}_{p}$, and it remains to show that $\langle 1,1,1,1\rangle=0$. Every element of a finite field is a sum of two squares; write $-2=x^{2}+y^{2}$. Then the linear span of $(x, y, 1,1)$ and $(1,1,-x,-y)$ in $\langle 1,1,1,1\rangle$ is Lagrangian.

C.7. Proposition B.23. Suppose $\left(A_{1}, q_{1}\right),\left(A_{2}, q_{2}\right)$ are quadratic modules. One sees immediately that

$$
\gamma\left(q_{1} \oplus q_{2}\right)=\gamma\left(q_{1}\right) \gamma\left(q_{2}\right) .
$$

We must check that $\gamma\left(q_{1}\right)=\gamma\left(q_{2}\right)$ whenever $\left(A_{1}, q_{1}\right)$ and $\left(A_{2}, q_{2}\right)$ are Witt equivalent. It suffices to show that $\gamma(q)=1$ whenever $(A, q)$ is a Witt-equivalent to 0 . For then equation (C.3) gives

$$
\gamma\left(q_{1}^{\circ}\right) \gamma\left(q_{2}\right)=\gamma\left(q_{1}^{\circ} \oplus q_{2}\right)=1 .
$$

In particular, $\gamma\left(q_{1}^{\circ}\right) \gamma\left(q_{1}\right)=1$. Therefore $\gamma\left(q_{1}\right)=\gamma\left(q_{2}\right)$. It also follows from (C.3) that $\gamma$ is a homomorphism. 
Suppose, then that $(A, q)$ contains a Lagrangian $L$, and let $S \subset A$ be a set of representatives of $A / L$. We find

$$
\begin{aligned}
\gamma(q) & =\frac{1}{\sqrt{|A|}} \sum_{a \in L} \sum_{s \in S} q(a+s,(a+s) / 2) \\
& =\frac{1}{\sqrt{|A|}} \sum_{a \in L} \sum_{s \in S} q(a, a / 2) q(a, s) q(s, s / 2) \\
& =\frac{1}{\sqrt{|A|}} \sum_{s \in S} q(s, s / 2) \sum_{a \in L} q(a, s) \\
& =q(0,0)=1 .
\end{aligned}
$$

The first equality is the definition, the second is bi-additivity, the third is because $L$ is isotropic, and the fourth uses that $\sum_{a \in L} \psi(q(a, s))=0$ unless $s \in L$.

\section{ACKNOWLEDGMENTS}

We would like to thank V. Drinfeld for introducing us to this project and sharing his notes and insights. In particular, the idea of using neighboring polarizations and results of Deligne and Quillen for defining the determinant are due to him. David Kazhdan and Ronny Hadani helped us understand the importance of this problem and encouraged us to pursue it. Last but not least, we would like to thank Travis Schedler for his interest in this paper and many stimulating conversations.

\section{REFERENCES}

[BD06] D. Boyarchenko and V. Drinfeld. A motivated introduction to character sheaves and the orbit method for unipotent groups in positive characteristic. arXiv:math/0609769v1, 2006.

[BLLV74] J. Barge, J. Lannes, F. Latour, and P. Vogel. $\Lambda$-sphères. Ann. Sci. École Norm. Sup. (4), 7:463-505 (1975), 1974. MR0377939 (51:14108)

[Del87] P. Deligne. Le déterminant de la cohomologie. In Current trends in arithmetical algebraic geometry (Arcata, Calif., 1985), volume 67 of Contemp. Math., pages 93-177. Amer. Math. Soc., Providence, RI, 1987. MR 902592 (89b:32038)

[Gai07] D. Gaitsgory. On de Jong's conjecture. Israel J. Math., 157:155-191, 2007. MR2342444 (2008j:14021)

[Gér77] P. Gérardin. Weil representation associated to finite fields. J. Algebra, 46(1):54-101, 1977. MR0460477 (57:470)

[GH07] S. Gurevich and R. Hadani. The geometric Weil representation. Selecta Math. (N.S.), 13(3):465-481, 2007. MR2383602 (2009e:11078)

[GH08] S. Gurevich and R. Hadani. Quantization of symplectic vector spaces over finite fields. J. Symplectic Geom. 7(4):475-502, 2009. MR2552002

[How73] R. Howe. On the character of Weil's representation. Trans. Amer. Math. Soc., 177:287298, 1973. MR0316633 (47:5180)

[How77] R. Howe. On representations of discrete, finitely generated, torsion-free, nilpotent groups. Pacific J. Math., 73(2):281-305, 1977. MR0499004 (58:16984)

[Kam05] M. Kamgarpour. Weil representations over finite fields. www.masoudkamgarpour.com/ Media Files/masterthesis.pdf, 2005.

[Kaz77] D. Kazhdan. Proof of Springer's hypothesis. Israel J. Math., 28(4):272-286, 1977. MR0486181(58:5959)

[Kir62] A. A. Kirillov. Unitary representations of nilpotent lie groups. Uspehi Mat. Nauk, 17:57110, 1962. MR0142001 (25:5396)

[Kir04] A. A. Kirillov. Lectures on the orbit method. American Math. Soc., 2004. MR2069175 (2005c:22001)

[Lam05] T.Y. Lam. Introduction to quadratic forms over fields, volume 67 of Graduate Studies in Mathematics. Amer. Math. Soc., 2005. MR2104929 (2005h:11075) 
[Lan05] S. Lang. Algebra, volume 211 of Graduate Text in Mathematics. Springer-Verlag, 2005. MR1878556 (2003e:00003)

[Laz54] M. Lazard. Sur les groupes nilpotents et anneaux de Lie. Ann. Sci. Eco. Norm. Sup., (31) 71:101-190, 1954. MR0088496 (19:529b)

[LP81] G. Lion and P. Perrin. Extension des représentations de groupes unipotents $p$-adiques. Calculs d'obstructions. In Noncommutative harmonic analysis and Lie groups (Marseille, 1980), volume 880 of Lecture Notes in Math., pages 337-356. Springer, Berlin, 1981. MR644839 (83h:22032)

[LV80] G. Lion and M. Vergne. The Weil representation, Maslov index and theta series, volume 6 of Progress in Mathematics. Birkhäuser Boston, Mass., 1980. MR573448 (81j:58075)

[Per81] P. Perrin. Représentations de Schrödinger, indice de Maslov et groupe metaplectique. In Noncommutative harmonic analysis and Lie groups (Marseille, 1980), volume 880 of Lecture Notes in Math., pages 370-407. Springer, Berlin, 1981. MR644841|(83m:22027)

[PPS00] R. Parimala, R. Preeti, and R. Sridharan. Maslov index and a central extension of the symplectic group. K-Theory, 19(1):29-45, 2000. MR.1740881(2001c:11053a)

[Qui73] D. Quillen. Higher algebraic K-theory. I. In Algebraic K-theory, I: Higher K-theories (Proc. Conf., Battelle Memorial Inst., Seattle, Wash., 1972), pages 85-147. Lecture Notes in Math., Vol. 341. Springer, Berlin, 1973. MR0338129(49:2895)

[RY02] Z. Reichstein and B. Youssin. A birational invariant for algebraic group actions. Pacific J. Math., 204(1):223-246, 2002. MR.1905199(2003c:14052)

[Tho06] T. Thomas. The Maslov index as a quadratic space. Math. Res. Lett., 5-6:985-999, 2006. MR2280792 (2007j:53093)

[Tho08] T. Thomas. The character of the Weil representation. J. LMS, 77(2):221-239, 2008. MR2389926 (2008k:11049)

[Wei64] A. Weil. Sur certains groupes d'opérateurs unitaires. Acta Math., 111:143-211, 1964. MR0165033(29:2324)

The University of British Columbia, Vancouver, Canada V6T $1 Z 2$

E-mail address: masoud@math.ubc.ca

The University of Edinburgh, Edinburgh, United Kingdom EH9 3JZ

E-mail address: t.thomas@ed.ac.uk 